\title{
On the traceably accurate voltage calibration of electrostatic accelerators
}

\author{
J.L. Colaux ${ }^{1}$, G. Terwagne ${ }^{2}$ and C. Jeynes ${ }^{1}$ \\ ${ }^{1}$ University of Surrey Ion Beam Centre, Guildford GU2 7XH, England \\ ${ }^{2}$ Université de Namur, Centre de Recherche en Physique Matière \& Rayonnement, \\ Laboratoire d'Analyses par Réactions Nucléaires (LARN), Namur B-5000, Belgium
}

\begin{abstract}
We describe in detail a calibration method for the terminal voltage of small accelerators used for ion beam analysis, with the elastic resonance of ${ }^{16} \mathrm{O}(\alpha, \alpha){ }^{16} \mathrm{O}$ at $3038 \mathrm{keV}$ as the intrinsic measurement standard. The beam energy relative to this resonance is determined with a precision around $300 \mathrm{eV}$ and an evaluated reproducibility of $1.0 \mathrm{keV}$. We show that this method is both robust and convenient, and demonstrate consistency with calibration relative to three other independent methods: using radioactive sources and using the resonant ${ }^{27} \mathrm{Al}(\mathrm{p}, \gamma)^{28} \mathrm{Si}$ and non-resonant ${ }^{16} \mathrm{O}(\mathrm{p}, \gamma){ }^{17} \mathrm{~F}$ direct capture reactions. We re-evaluate the literature and show that the peak in the crosssection function is at $3038.2 \pm 3.1 \mathrm{keV}$. By comparing the results obtained with ${ }^{16} \mathrm{O}(\alpha, \alpha){ }^{16} \mathrm{O}$ to the other calibration methods we show that this uncertainty can be reduced to $1.0 \mathrm{keV}$.
\end{abstract}

\section{Keywords}

IBA, PIXE RBS, EBS, NRA, elastic scattering cross-section, Rutherford backscattering, astrophysics, accurate analysis, CCQM, quantity of material

A version of this paper was accepted by Nuclear Instruments \& Methods B, 18th February 2015, and subsequently published (Nucl. Instruments \& Methods B, 2015, http://dx.doi.org/10.1016/i.nimb.2015.02.048) 


\section{Introduction}

The energy calibration of the small accelerators used for ion beam analysis (IBA) has long been well and extensively treated in the literature. However, the standard calibration points such as the important ${ }^{27} \mathrm{Al}(p, \gamma){ }^{28} \mathrm{Si}$ resonant reaction at $992 \mathrm{keV}$ [1] or neutron thresholds such as the ${ }^{7} \mathrm{Li}(p, n)^{8} \mathrm{Be}$ at $1881 \mathrm{keV}$ [2] are mostly for a proton beam at various energies. Analysts using an alpha beam (for an enhanced depth resolution) have not conveniently been able to verify their energy calibration. Other methods such as nonresonant proton capture [3] or absolute velocity gauge (a time-of-flight method) [4] can directly give the beam energy, but these are unsuitable for routine use because the crosssections are so low and because an independent absolute calibration of the gamma spectroscopy system is required for the first method and complex instrumentation is required for the second.

Rutherford backscattering spectrometry (RBS) spectra are notorious for being similar whatever the beam energy, since the kinematical factor does not depend on energy. However, elastic (non-Rutherford) backscattering (EBS) spectra are very sensitive to energy, provided there is a resonance at an appropriate energy in the scattering crosssection function. It turns out that the $3038 \mathrm{keV}$ resonance in the ${ }^{16} \mathrm{O}(\alpha, \alpha)^{16} \mathrm{O}$ reaction is: (a) quite narrow at about $10 \mathrm{keV}$; (b) very strong at up to $29 \times$ Rutherford for backscattering angles; (c) at a very convenient energy for most small accelerators used for ion beam analysis (IBA); and (d) evaluated [5], that is, fitted to a nuclear model consistent with all measured scattering cross-section data as well as other relevant nuclear data. These evaluated data are available and valid at any backscattering angle [6].

The Surrey Ion Beam Centre has recently made routine use of this elastic ${ }^{16} \mathrm{O}(\alpha, \alpha){ }^{16} \mathrm{O}$ reaction resonance at $3038 \mathrm{keV}$. We will show that the beam energy can be determined at remarkably good precision, and an accuracy (estimated by a full uncertainty budget) that is dominated by the accuracy with which the resonance energy is known. We will show that from evaluating the literature that this resonance energy is at $3038.2 \pm 3.1 \mathrm{keV}$, and SigmaCalc-v2.0 [6] has been adjusted to give this value.

This calibration method has been validated at both universities of Surrey and Namur by comparison to other calibration methods: namely, the ${ }^{27} \mathrm{Al}(p, \gamma){ }^{28} \mathrm{Si}$ resonant reaction [1] \& the non-resonant ${ }^{16} O(p, \gamma)^{17} F$ proton capture [3] at Surrey, and the use of a triple-alpha source $[7,8]$ at Surrey \& Namur. We will show that these new measurements enable us to significantly reduce the uncertainty on the position of this ${ }^{16} \mathrm{O}(\alpha, \alpha){ }^{16} \mathrm{O}$ resonance.

In a companion work [9], we describe in detail an extension to this method which also very accurately calibrates the pulse-height spectrometry system, taking into account 
the non-linearity of the detectors known as the "pulse-height defect" (PHD) $[8,10]$, that is, the energy losses in the entrance electrode, the detector dead layer and non-ionising (nuclear) processes that are not registered in the detected pulse-height. This builds on an inter-laboratory comparison which also discussed RBS in detail, including a close discussion of the formalism and a variety of non-linear effects [11], and a longitudinal study in which the validity of a critically constructed uncertainty budget was demonstrated [12].

\section{Experimental details}

\section{University of Surrey (IBC)}

All the measurements were performed with the $2 \mathrm{MV}$ HVEE Tandetron ${ }^{\mathrm{TM}}$ accelerator installed at the IBC [13]. The beam energy is controlled using feedback from the generating voltmeter (GVM) monitoring the tandem terminal voltage.

For the ${ }^{16} \mathrm{O}(\alpha, \alpha){ }^{16} \mathrm{O}$ calibration method, EBS spectra were acquired using the 6 -axis goniometer which allows air-lock handling of $100 \mathrm{~mm}$ wafers without breaking vacuum [13]. Two standard semiconductor diode detectors were used at backscattering angles of $173.4^{\circ}$ (DetA) and $148.8^{\circ}$ (DetB), measured with an accuracy of $0.2^{\circ}$ using the goniometer with an in-line laser. The solid angles of detection were 0.9 and $2.1 \mathrm{msr}$ for DetA and DetB, respectively. Standard analogue electronics were used for pulse-height amplification and measurement with successive-approximation ( $6 \mu$ s conversion time) ADCs. The shaping amplifiers have a shaping time of about 500 ns, and implement a pulse-pileup inspection circuit with a time resolution also of about 500 ns: the ADCs were gated to reject detected pileup events. The ADC electronic zero ("offset") was measured directly using an electronic pulser: that is, electronic pulses of various heights (measured with a storage oscilloscope) are recorded by the pulse-height spectrometry system. The offset (in channels) is determined from a linear regression of the pulse-height (in volts) and the pulse position (in channel number). The offset in keV follows from knowing the electronic gain of the pulseheight spectrometry system (keV/ch).

A series of 9 spectral pairs (from the two detectors), acquired close to the $3038 \mathrm{keV}$ resonance (typically, from 3040 to $3088 \mathrm{keV}$ by $6 \mathrm{keV}$ steps), were obtained from the standard "Au/Ni/SiO $2 / \mathrm{Si}$ " sample [14]. The RBS spectra were fitted using the DataFurnace code [15] with computation engine NDFv9.6a and WiNDFv9.3.76 [16]. This code implements Andersen screening [17], SRIM-2003 stopping powers [18] (note that the latest SRIM [19] is not materially different), Molodtsov \& Gurbich pileup correction [20], and also the pulse-height defect (PHD) correction of Pascual-Izarra \& Barradas [10] which uses Lennard's calculation of the non-ionising energy loss [8]. The channelled substrate signals 
were fitted using an ad-hoc cubic polynomial correction to the scattering cross-section of $\mathrm{Si}$, discussed in detail by Barradas et al [21]. $\mathrm{SiO}_{2}$ electronic stopping is not given accurately by Bragg's rule; instead the molecular stopping measured by Lennard et al [22] is used. NDF uses both global and local minimisation algorithms, usually of a $\chi^{2}$ function, although a "robustified" $\chi^{2}$ function [23] can also be used since $\chi^{2}$ can be mathematically too badly behaved far from the minimum. Of course, any function representing goodness of fit can be used as the "objective function" [24] for minimisation: we choose the $\chi^{2}$ function for convenience.

The same experimental setup was used for the triple-alpha $\left({ }^{239} \mathrm{Pu}^{241} \mathrm{Am}^{244} \mathrm{Cm}\right)$ source calibration method. Spectra were obtained from a microscope cover slip for a terminal voltage set at $1478.0 \mathrm{kV}$. During this analysis, the triple-alpha source was sitting just next to the sample $\mathrm{SiO}_{2} / \mathrm{Si}$ sample for simultaneously recording the characteristic alpha peaks produced by the ${ }^{239} \mathrm{Pu},{ }^{241} \mathrm{Am} \&{ }^{244} \mathrm{Cm}$ decays.

For the ${ }^{27} \mathrm{Al}(\mathrm{p}, \gamma)^{28} \mathrm{Si}$ calibration method, the excitation curve was measured stepping the terminal voltage between $479 \& 481 \mathrm{kV}$ in $0.1 \mathrm{kV}$ steps. The high energy gamma rays emitted by the ${ }^{28} \mathrm{Si}^{*}$ decay were collected using a Nal(TI) detector (diameter $50 \mathrm{~mm}$; length $50 \mathrm{~mm}$ ) for a constant collected charge of $2 \mu \mathrm{C}$. The sample was a fresh piece of Al foil.

For the non-resonant ${ }^{16} \mathrm{O}(\mathrm{p}, \mathrm{\gamma})^{17} \mathrm{~F}$ calibration method, we used a $1 \mu \mathrm{m}$ thermal oxide grown on $100 \mathrm{~mm} \mathrm{Si} \mathrm{wafer} \mathrm{in} \mathrm{combination} \mathrm{with} \mathrm{a} \mathrm{proton} \mathrm{beam} \mathrm{energy} \mathrm{set} \mathrm{around}$ $1650 \mathrm{keV}$. The gamma rays emitted by the non-resonant radiative proton capture were detected with a high purity Germanium (HPGe) detector calibrated with the environmental ${ }^{40} \mathrm{~K}$ line (1460.9 keV), the $\mathrm{e}^{-}-\mathrm{e}^{+}$annihilation (511 keV) and the ${ }^{60} \mathrm{Co}$ radioactive source (1173.2 and $1332.5 \mathrm{keV}$ ). The gamma ray spectrum was for a collected charge of $1.2 \mathrm{mC}$ (with a beam current of about $1 \mu \mathrm{A}$ ).

\section{University of Namur (LARN)}

RBS measurements were performed with the 2 MV HVEE Tandetron ${ }^{\mathrm{TM}}$ accelerator installed at the LARN. Three standard semiconductor diode detectors were used at backscattering angles of $170^{\circ}$ (DetC), $165^{\circ}$ (DetD) and $135^{\circ}$ (DetE), with an accuracy of $0.5^{\circ}$ derived from the machining precision of the RBS chamber lid holding the detectors. The solid angles of detection were 3.1, 3.0 and 3.0 msr for DetC, DetD and DetE, respectively. The beam energy is controlled using feedback from the GVM monitoring the tandem terminal voltage. Experimental spectra were recorded using standard analogue electronics together with an ultra-fast analog-to-digital converter (ADC FastComtec 7072). The amplifiers have a shaping time of about 500 ns, and implement a pulse-pileup inspection 
circuit with a time resolution also of about 500 ns. For each detector, the actual dead layer was derived from the comparison of the 3-alpha source $\left({ }^{239} \mathrm{Pu}^{241} \mathrm{Am}^{244} \mathrm{Cm}\right)$ spectra recorded with the detector normal to the source and tilted at 60 degrees to it [8].

The ${ }^{16} \mathrm{O}(\alpha, \alpha)^{16} \mathrm{O}$ calibration method described in this work was used to determine the GVM calibration factor (i.e. the relationship between the indicated and the actual terminal voltage) of the accelerator; then the incident alpha beam was set at an indicated energy of $3.000 \mathrm{MeV}$. Two sets of three spectra were obtained from two different samples (namely, $\mathrm{Ta}_{2} \mathrm{O}_{5} / \mathrm{C}$ and $\mathrm{SiO}_{2} / \mathrm{Si}$ ) along with the triple-alpha $\left({ }^{239} \mathrm{Pu}^{241} \mathrm{Am}^{244} \mathrm{Cm}\right.$ ) source sitting just next to the sample $\mathrm{Ta}_{2} \mathrm{O}_{5} / \mathrm{C}$ (or $\mathrm{SiO}_{2} / \mathrm{Si}$ ). The particle spectra were fitted using the DataFurnace code as for Surrey.

\section{${ }^{16} \mathrm{O}(\alpha, \alpha){ }^{16} \mathrm{O}$ energy calibration method}

Figure 1 shows a typical spectrum from the "standard" $\mathrm{Au} / \mathrm{Ni} / \mathrm{SiO}_{2} / \mathrm{Si}$ sample used to determine the gain of the spectroscopic electronics [14] in which the $3038 \mathrm{keV}$ ${ }^{16} \mathrm{O}(\alpha, \alpha){ }^{16} \mathrm{O}$ resonance is prominent in the $330 \mathrm{~nm} \mathrm{SiO}{ }_{2}$ layer. The positions of peaks and edges in these spectra are extremely well determined independently of the energy resolution of the system, given sufficient signal. This was shown explicitly by Jeynes et al $[14,25]$, and the $\chi^{2}$ minimisation used by DataFurnace is mathematically equivalent, obtaining the same precision.

Given the backscattering angle and PHD of the detector, the incident beam energy can be accurately derived from the ${ }^{16} \mathrm{O}$ signal shape; the relative position of the peaks or edges (for $\mathrm{Au}, \mathrm{Ni}, \mathrm{Si}, \mathrm{O}$ ) also enables the determination of the electronic gain and PHD. The effect of the PHD is not the same as the effect of a simple offset, since the latter is strictly independent of energy but the energy loss in the dead layer varies markedly with energy $[8,26]$. It is only because the dead layer is rather thin that this large difference has only a small effect on the calculated spectrum, but the correct assignment of PHD is crucial to accurately determining the electronic gain of the spectrometry system and obtain good fitting of the whole spectrum [11].

The spectra collected around 3.0 MeV are nevertheless rather insensitive to the PHD value used in the simulations. This is because of the reduction of the energy lost within the dead layer due to relatively high energy of the backscattered particles (the oxygen signal is still around $1 \mathrm{MeV}$ ). Consequently, we have estimated that the PHD determination from the dataset collected around $3 \mathrm{MeV}$ cannot be done at better than about $20 \%$. Equally, the comparison between the experimental and fitted offset values is of limited use for assessing the PHD effect since it is difficult to measure the offset with an uncertainty smaller than about $10 \mathrm{keV}$. Consequently, the electronic gain derived from this 
analysis cannot be determined with an accuracy much better than about $0.4 \%$ even when acquiring a series of spectra at various incident energies around the resonance (i.e. typically from 3040 to $3088 \mathrm{keV}$ by $6 \mathrm{keV}$ steps). This is unfortunately not quite good enough for high accuracy RBS measurements [12,27], which is why a full method for reducing this uncertainty down to about $0.1 \%$ is given in a companion paper [9].

Figure 1 shows one spectrum at one energy: we collected 9 pairs of spectra from two detectors at 9 energies near $3 \mathrm{MeV}$ (see Table 1). In the present work, we wish to extract from this set of 9 spectral pairs: a) the beam energy, b) the dead layer of the detector and c) the electronic gain of the detection channel. These parameters are all correlated, as Siketić et al [28] have shown in detail; an iterative procedure (see flow diagram in Figure 2) must be used to converge on the optimum values. Therefore, extracting these parameters from such a dataset requires one to use a code incorporating automated features. We used DataFurnace (NDF), which is one of the "new generation" codes [29], and also has extensive fitting functions. NDF is also able to accurately simulate spectra involving strong and sharp EBS resonances, a non-trivial calculation in principle involving double integrations [30].

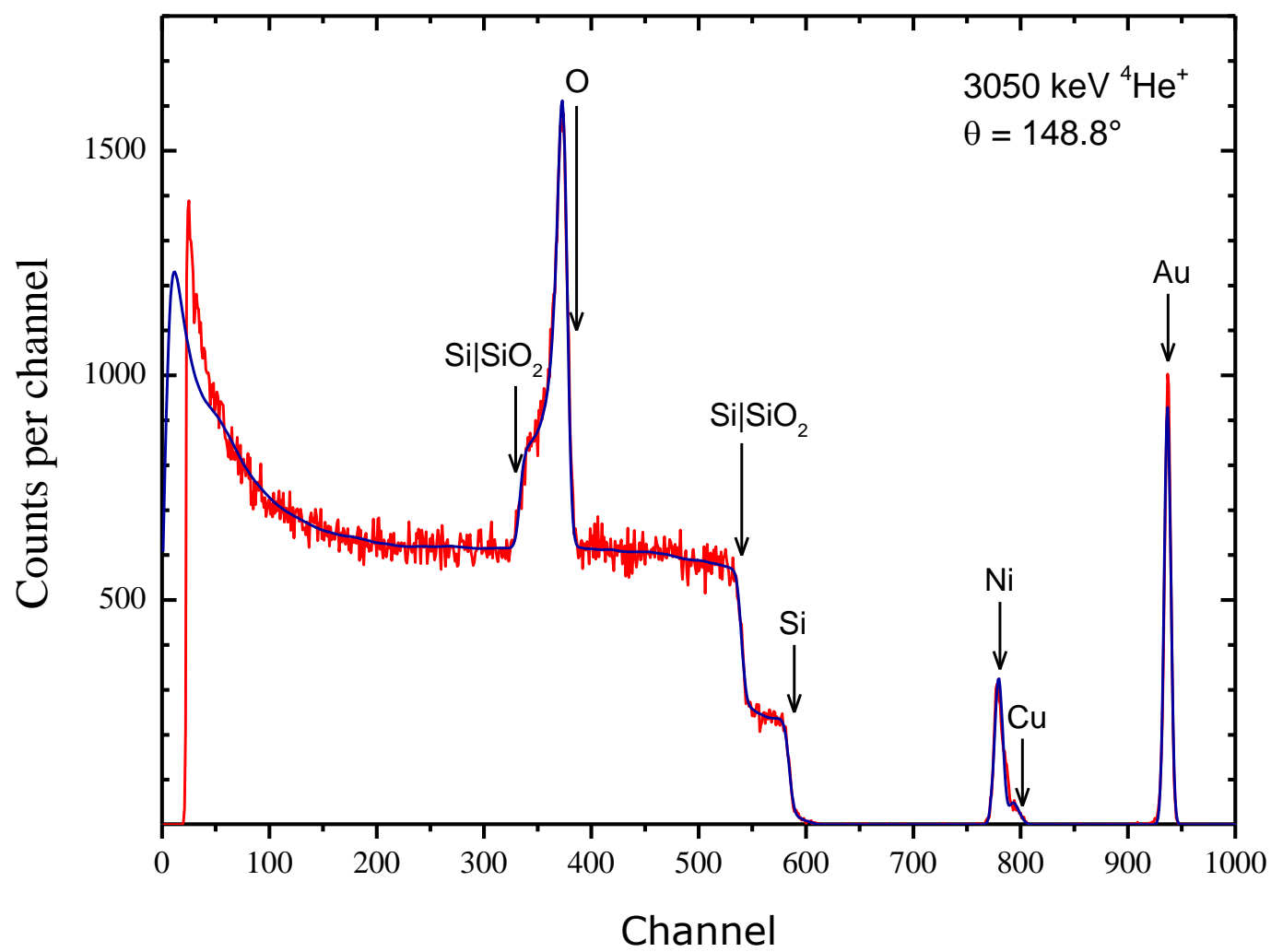

Figure 1: Pulse-height spectrum (red) from the standard calibration sample, with fit (blue). This sample has a nominal structure $(10,20,2200)$ TFU of $\left(\mathrm{Au}, \mathrm{Ni}, \mathrm{SiO}_{2}:\right.$ TFU $\equiv$ "Thin Film Unit" $\equiv 10^{15}$ atoms $\left./ \mathrm{cm}^{2}\right)$. This is equivalent to $1.7,2.2$ and $333 \mathrm{~nm}$ at full bulk density. The collected charge is about $10 \mu \mathrm{C}$. The Ni actually has about $10 \% \mathrm{Cu}$, as confirmed by PIXE. There is (slight) channelling on the Si substrate fitted by an ad hoc cubic polynomial: higher order terms are needed at low energies because of multiple scattering and other effects. Elemental edges are shown, together with the interface positions in the $\mathrm{Si}$ and $\mathrm{O}$ signals. 


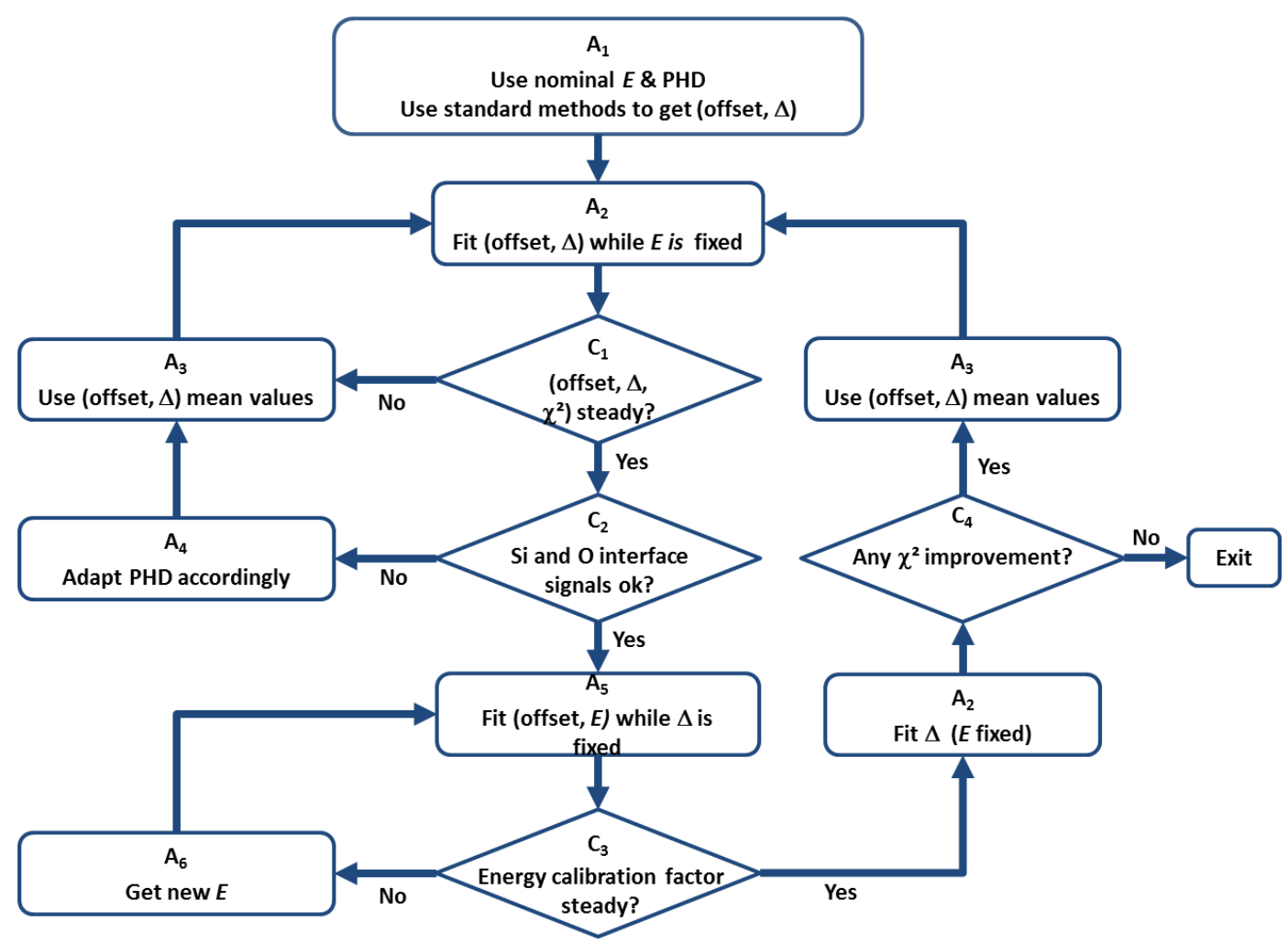

Figure 2: Flow diagram of the energy calibration procedure. $E$ is the incident beam energy; offset and $\Delta$ are the electronic offset and gain of the pulse-height spectrometry system; $\chi^{2}$ is the chi-squared function calculated for each spectral fit. The output of this procedure is the gain, offset, pulse-height defect (PHD), and set of beam energies for each detector.

Figure 2 shows a flow diagram of the procedure used to determine the GVM calibration factor. Considering the steps in detail :-

$A_{1}$ The nominal incident beam energies $(E)$ are derived from previous calibrations done with this or another calibration method. In any case, since the energy of the incident beam can be precisely derived from the ${ }^{16} \mathrm{O}$ peak for a given scattering angle and PHD, prior knowledge about the accelerator energy calibration is not strictly needed. The nominal PHD value may come from the datasheet of the detector; from previous calibrations or from direct measurement using a triple-alpha source. When no information is available, the PHD value is roughly determined by fixing the electronic offset at the value directly measured with an electronic pulser.

$A_{2}$ The whole dataset is fitted using DataFurnace, that is, all spectra are automatically fitted one after the other where the incident energy and PHD are fixed to nominal values and the electronic gain ( $\pm 2 \%$ ) and offset ( \pm 10 channels) are fitted to minimise the chi-squared $\left(\chi^{2}\right)$ of each spectrum. Other parameters such as the collected charge ( $\pm 20 \%)$, the detector resolution $( \pm 10 \mathrm{keV})$ and the sample depth profile are also enabled to vary during the $\chi^{2}$ minimisation. 
We should comment that the detector resolution is fitted because $\chi^{2}$ minimisation is extremely sensitive to the shape of edges and peaks, and if the instrumental function is not correct the positions of both edges and peaks can be heavily distorted. The purpose is to use these features of the spectra to determine the experimental parameters; it is of the essence to avoid such distortions.

$C_{1}$ The results of the fitting procedure $\left(A_{2}\right)$ are exported to a spreadsheet where the mean and standard deviation (SD) of each fitted parameter are calculated and recorded for tracking their trend as a function of fitting iteration number (see Figure 3). If the (offset, $\Delta, \chi^{2}$ ) mean values have not reached a steady state or if their SD is not satisfying, these values are used as inputs $\left(A_{3}\right)$ for performing the next fitting iteration $\left(A_{2}\right)$.

$\mathrm{C}_{2}$ When the criterion $\mathrm{C}_{1}$ is satisfied the analyst closely looks at the silicon ( $\left.\mathrm{Si} \& \mathrm{Si} \mid \mathrm{SiO}_{2}\right)$ and oxygen ( $\mathrm{O} \& \mathrm{Si} \mid \mathrm{SiO}_{2}$ ) interface signals (see Figure 1 ). If all these edges are not properly fitted for each spectrum, the PHD value is accordingly adjusted $\left(A_{4}\right)$ and a new iteration is performed $\left(A_{3}+A_{2}\right)$.

$A_{5}$ When the criterion $C_{2}$ is satisfied the electronic gain is fixed at the mean value obtained in the latest iteration, and the incident energy is then fitted to minimise the $\chi^{2}$ for each spectrum. Other parameters such as the electronic offset, the collected charge, the detector resolution and the sample depth profile are also varied as before during the $\chi^{2}$ minimisation.

We should comment that the $O$ signal shape near the resonance is exquisitely sensitive to beam energy and surprisingly sensitive to scattering angle, and that NDF interpolates the evaluated resonance function both for angle and for energy. This procedure depends on the scattering angle to be accurately known. NDF also supports $\chi^{2}$ fitting for angle, but determining and validating the angle is done separately.

$C_{3}$ The results obtained by $A_{5}$ are exported to a spreadsheet where the mean GVM calibration factor is calculated and recorded (see Figure 3 ). If this factor has not reached a steady state, the nominal incident energies are recalculated $\left(A_{6}\right)$ and a new energy fitting iteration $\left(A_{5}\right)$ is performed.

$C_{4}$ When the criterion $C_{3}$ is satisfied we switch again to $A_{2}$ fitting procedure (Energy fixed; offset and gain fitted). If no significant improvement of the mean $\chi^{2}$ value is obtained, the entire calibration procedure is completed. Otherwise, the mean electronic offset and gain are used as inputs $\left(A_{3}\right)$ for the next iteration $\left(A_{2}\right)$.

It should be noted that both oxygen and silicon edges at the $\mathrm{Si} \mid \mathrm{SiO}_{2}$ interface are sensitive to errors existing in the $\mathrm{SiO}_{2}$ stopping power function. Since the exit path length is quite different for the two detectors, we separately fit the spectra recorded by DetA \& DetB, so that any error in the stopping power function can be compensated by adapting the nominal $\mathrm{SiO}_{2}$ thickness used for the simulation. 


\section{Results}

\section{University of Surrey (IBC)}

Fitting the whole dataset (18 spectra: 2 detectors, 9 energies) with DataFurnace and exporting the results to a spreadsheet (this is step $A_{2}$ in Figure 2), one can obtain a table similar to Table 1 where the standard error at 99\% of confidence (SE99) is given by:

$$
S E 99=\left(S D \times \sqrt{\frac{N-1}{\operatorname{Chiinv}\left(1-\frac{1-\alpha}{2} ; N-1\right)}}\right) / \sqrt{N-1} \quad \text { Eq.1 }
$$

where $\boldsymbol{S D}$ is the standard deviation of the $\boldsymbol{N}$ measurements, $\boldsymbol{\alpha}$ is the interval of confidence (i.e. 99\%) and Chiinv is a function returning the inverse of the right-tailed probability of a chi-squared distribution.

Logging the mean value of the fitted parameters (electronic gain, offset, PHD and $\left.\chi^{2}\right)$ after each iteration $\left(A_{2}\right.$ in Figure 2), one can obtain the trend charts shown in Figure 3: Evolution of the fitted parameters with iteration number. See Error! Reference source not found.2 for the gain calibration method. The fitted parameters are: electronic gain, offset, PHD and $\chi^{2}$. In the analysis shown, the electronic offset measured directly using an electronic pulser was found to be $(-8.4 \pm$ 14.0) $\mathrm{keV}$ for DetA and $(19.2 \pm 13.5) \mathrm{keV}$ for DetB. The standard error on these values is quite large due to the limited accuracy for the pulse-height measurement available with the oscilloscope.

and used for assessing the criterion $\mathrm{C}_{1}$. In this example, only a couple of iterations are needed for reaching a steady state of all fitted parameters because of prior knowledge available about the accelerator and the detectors: the GVM calibration factor is well under control as discussed later, and the PHD values are known from previous analyses. The criteria $C_{1}$ and $C_{2}$ are fulfilled after 6 iterations where we switched from the electronic gain fitting $\left(A_{2}\right)$ to the beam energy fitting $\left(A_{5}\right)$. The results of this iteration are exported in a table similar to Table 2, where the GVM calibration factor is calculated according to

$$
a=\frac{E_{p}-e V_{e x t}+E_{s t r}}{(1+q) e V_{t}} \quad \text { Eq.2 }
$$

where

- $\quad \boldsymbol{E}_{\boldsymbol{p}}$ is the energy of the accelerated particle $\left({ }^{4} \mathrm{He}^{+}\right.$in this case);

- $\quad \boldsymbol{e}$ is the electron charge;

- $\quad V_{\text {ext }}$ is the extraction voltage applied to the ion source (assuming singly charged negative ions are extracted);

- $\quad \boldsymbol{q}$ is the charge state of the accelerated particles ( $q=1$ in this case);

- $\boldsymbol{a}$ is the accelerator (or GVM) calibration factor such that the true terminal voltage is $\boldsymbol{a} . \boldsymbol{V}_{\boldsymbol{t}}$;

- $\quad V_{t}$ is the indicated voltage set by the user on the high terminal voltage controller;

- $\quad \boldsymbol{E}_{\text {str }}$ is the energy loss of the accelerated particles going through the stripper channel of the tandem accelerator. 
The incident energies are then recalculated according to the new GVM calibration factor $\left(A_{6}\right)$ which leads to significant improvement of $\chi^{2}$ (iteration \#7), with the final results shown in Table 2.

For validation purposes, the results obtained with the present ${ }^{16} \mathrm{O}(\alpha, \alpha){ }^{16} \mathrm{O}$ calibration method were compared with three other calibration methods: namely, the resonant ${ }^{27} \mathrm{Al}(\mathrm{p}, \gamma)^{28} \mathrm{Si}$ and non-resonant ${ }^{16} \mathrm{O}(\mathrm{p}, \gamma){ }^{17} \mathrm{~F}$ proton capture reactions, and measurements with the triple-alpha source.

For the well-known ${ }^{27} \mathrm{Al}(\mathrm{p}, \gamma)^{28} \mathrm{Si}$ calibration method, we used a Nal(TI) scintillation detector for measuring the high energy gamma count rate (in the window $9.5-12.5 \mathrm{MeV}$ ) as a function of the incident beam energy. Figure 3 shows the excitation curve, fitted with a Boltzmann function whose centre is $479.92 \pm 0.01 \mathrm{kV}$. Since the energy of the resonance is known to be $991.90 \pm 0.04 \mathrm{keV}$ [1], the GVM calibration factor can be derived from this value using Eq.2. We found $1.0075 \pm 0.0005$, assuming $0.07 \mathrm{keV}$ as the estimated energy loss in the stripper $\left(E_{s t r}\right)$ and using the nominal extraction voltage $\left(V_{\text {ext }}\right)$ of $24.9 \mathrm{kV}$. The uncertainty on the GVM calibration factor is derived in the "Uncertainty Budget" section.

\begin{tabular}{|c|c|c|c|c|c|c|}
\hline Detector & $\begin{array}{c}\text { Indicated terminal } \\
\text { voltage } \\
\mathrm{kV}\end{array}$ & $\begin{array}{c}\text { Detector resolution } \\
\text { FWMH } \\
\text { keV }\end{array}$ & $\begin{array}{c}\text { Beam energy } \\
\mathrm{keV}\end{array}$ & $\begin{array}{c}\text { Electronic gain } \\
\mathrm{keV} / \mathrm{ch}\end{array}$ & $\begin{array}{c}\text { Electronic } \\
\text { offset } \\
\text { keV }\end{array}$ & $\chi^{2}$ \\
\hline \multirow{9}{*}{ 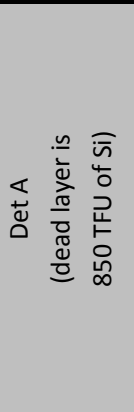 } & 1500 & 22.0 & 3041.7 & 3.3763 & -4.2 & 1.19 \\
\hline & 1503 & 22.1 & 3047.7 & 3.3762 & -4.4 & 1.28 \\
\hline & 1506 & 22.9 & 3053.8 & 3.3780 & -5.6 & 1.44 \\
\hline & 1509 & 23.1 & 3059.8 & 3.3769 & -4.6 & 1.38 \\
\hline & 1512 & 23.3 & 3065.9 & 3.3778 & -4.7 & 1.15 \\
\hline & 1515 & 23.5 & 3071.9 & 3.3777 & -4.5 & 0.92 \\
\hline & 1518 & 23.6 & 3078.0 & 3.3782 & -4.4 & 1.26 \\
\hline & 1521 & 24.1 & 3084.0 & 3.3788 & -4.5 & 1.24 \\
\hline & 1524 & 24.0 & 3090.0 & 3.3780 & -4.5 & 1.29 \\
\hline \multicolumn{2}{|r|}{ Average } & 23.2 & & 3.3775 & -4.6 & 1.24 \\
\hline \multicolumn{2}{|r|}{ SD } & $3.2 \%$ & & $0.026 \%$ & 0.4 & 0.15 \\
\hline \multicolumn{2}{|r|}{ SE99 (Eq.1) } & $2.8 \%$ & & $0.022 \%$ & & \\
\hline \multirow{9}{*}{ 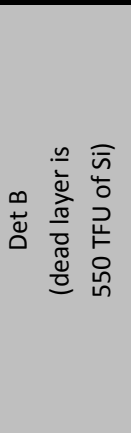 } & 1500 & 29.0 & 3041.7 & 3.3258 & 28.6 & 1.67 \\
\hline & 1503 & 28.5 & 3047.7 & 3.3264 & 28.3 & 1.71 \\
\hline & 1506 & 28.3 & 3053.8 & 3.3271 & 28.6 & 1.67 \\
\hline & 1509 & 28.5 & 3059.8 & 3.3271 & 28.6 & 1.62 \\
\hline & 1512 & 29.1 & 3065.9 & 3.3282 & 28.6 & 1.34 \\
\hline & 1515 & 28.8 & 3071.9 & 3.3278 & 28.7 & 1.40 \\
\hline & 1518 & 30.3 & 3078.0 & 3.3292 & 28.7 & 1.51 \\
\hline & 1521 & 30.1 & 3084.0 & 3.3289 & 28.9 & 1.66 \\
\hline & 1524 & 30.1 & 3090.0 & 3.3284 & 28.9 & 1.65 \\
\hline \multicolumn{2}{|r|}{ Average } & 29.2 & & 3.3277 & 28.7 & 1.58 \\
\hline \multicolumn{2}{|r|}{ SD } & $2.7 \%$ & & $0.034 \%$ & 0.2 & 0.13 \\
\hline \multicolumn{2}{|r|}{ SE99 (Eq.1) } & $2.3 \%$ & & $0.030 \%$ & & \\
\hline
\end{tabular}

Table 1: Final fitted values for gain, offset, resolution and $\chi^{2}$. See the energy calibration procedure described in Figure 2. 


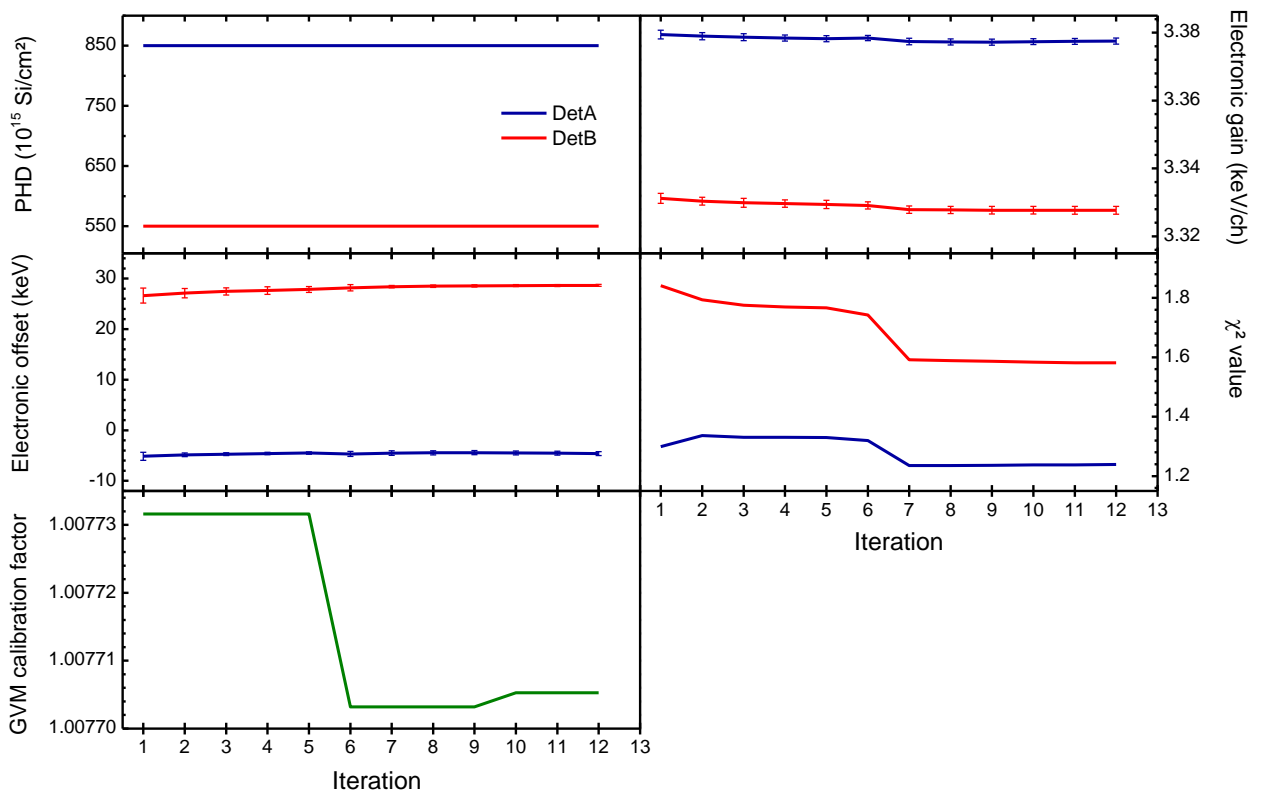

Figure 3: Evolution of the fitted parameters with iteration number. See Error! Reference source not found.2 for the gain calibration method. The fitted parameters are: electronic gain, offset, PHD and $\chi^{2}$. In the analysis shown, the electronic offset measured directly using an electronic pulser was found to be $(-8.4 \pm 14.0) \mathrm{keV}$ for DetA and $(19.2 \pm 13.5)$ $\mathrm{keV}$ for DetB. The standard error on these values is quite large due to the limited accuracy for the pulse-height measurement available with the oscilloscope.

\begin{tabular}{|c|c|c|c|c|c|}
\hline \multirow{2}{*}{$\begin{array}{c}\text { Indicated terminal voltage } \\
\mathrm{kV}\end{array}$} & $\begin{array}{c}\text { Det A } \\
(\mathrm{keV})\end{array}$ & $\begin{array}{c}\text { Det B } \\
(\mathrm{keV})\end{array}$ & $\begin{array}{c}\text { Average } \\
(\mathrm{keV})\end{array}$ & $\begin{array}{c}\text { Ratio } \\
\text { Det A / Det B }\end{array}$ & $\begin{array}{c}\text { GVM } \\
\text { calibration } \\
\text { factor }\end{array}$ \\
\hline 1500.0 & 3042.9 & 3042.9 & 3042.9 & 1.00000 & 1.00779 \\
\hline 1503.0 & 3049.1 & 3048.9 & 3049.0 & 1.00007 & 1.00781 \\
\hline 1506.0 & 3054.4 & 3054.8 & 3054.6 & 0.99987 & 1.00766 \\
\hline 1509.0 & 3060.7 & 3060.7 & 3060.7 & 1.00000 & 1.00768 \\
\hline 1512.0 & 3066.6 & 3066.7 & 3066.7 & 0.99997 & 1.00765 \\
\hline 1515.0 & 3072.9 & 3073.1 & 3073.0 & 0.99993 & 1.00775 \\
\hline 1518.0 & 3078.9 & 3079.0 & 3079.0 & 0.99997 & 1.00772 \\
\hline 1521.0 & 3084.8 & 3085.2 & 3085.0 & 0.99987 & 1.00772 \\
\hline 1524.0 & 3090.9 & 3091.1 & 3091.0 & 0.99994 & 1.00771 \\
\hline \multicolumn{3}{|c|}{ Average } \\
\hline \multicolumn{3}{|c|}{ SD } & & 0.99996 & 1.00772 \\
\hline
\end{tabular}

Table 2: Fitting the beam energy for a fixed electronic gain and PHD (Surrey data). The GVM calibration factor is derived from Eq. 2 using the mean fitted energy, an extraction voltage of $20.0 \mathrm{kV}$ and assuming $0.47 \mathrm{keV}$ as the estimated energy loss in the stripper. 


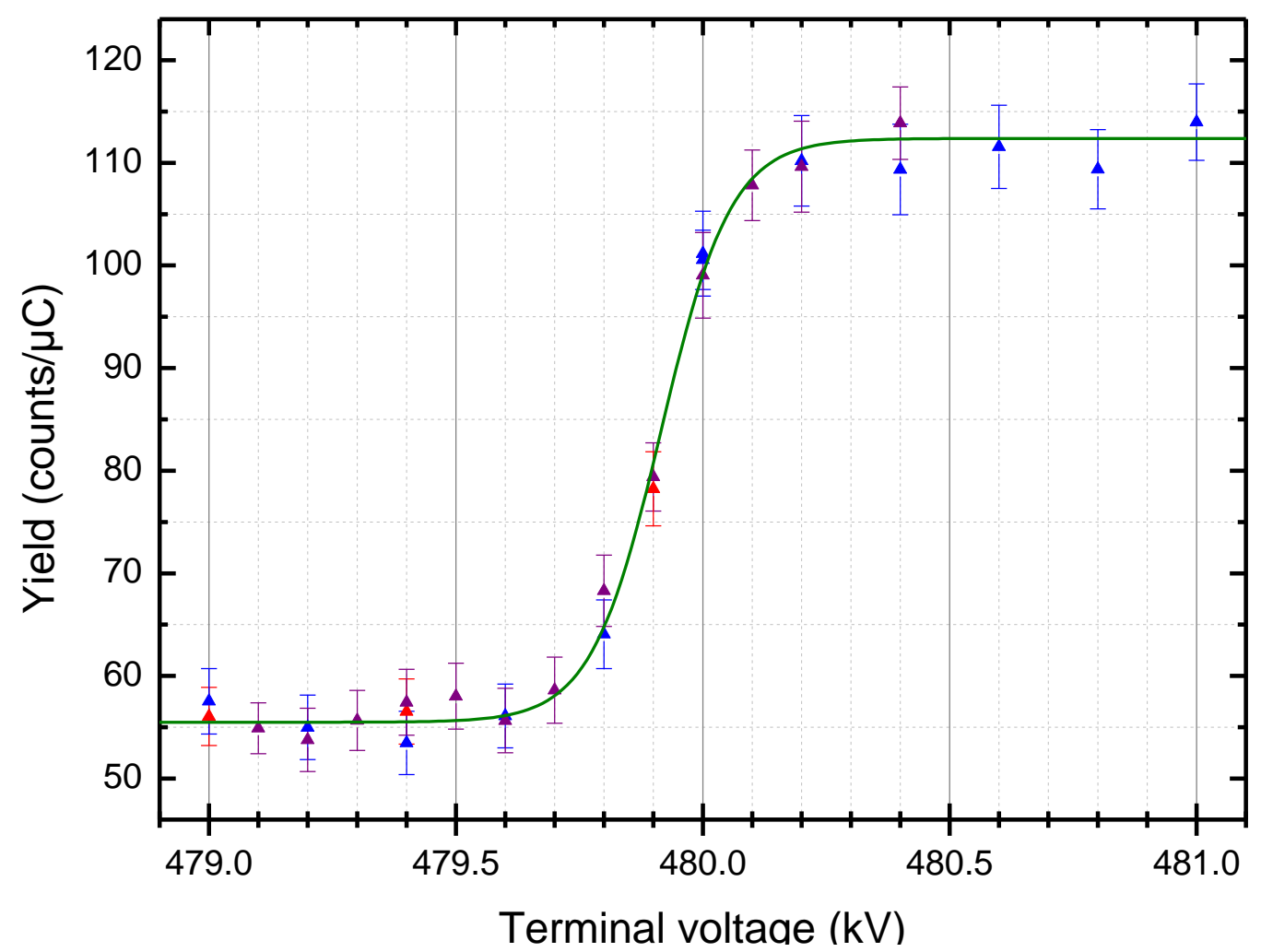

Figure 3: Excitation curve of the ${ }^{27} \mathrm{Al}(\mathrm{p}, \gamma)^{28} \mathrm{Si}$ direct capture. This was measured (Jan14) using Nal(TI) scintillator detector to count the high energy gamma rays $(9.5-12.5 \mathrm{MeV})$ and fitted by a Boltzmann curve (in green). The centre of the Boltzmann curve is $479.916 \pm 0.005 \mathrm{kV}$. Different colour data points are repeated measurement series in different direction, so that the precision setting and stability of the GVM are clearly excellent.

For the ${ }^{16} \mathrm{O}(\mathrm{p}, \mathrm{\gamma})^{17} \mathrm{~F}$ calibration method, the energy of the gamma rays $\left(\boldsymbol{E}_{\boldsymbol{\gamma}}\right)$ emitted by the non-resonant radiative proton capture were measured with a high purity Germanium (HPGe) detector. Since $\boldsymbol{E}_{\boldsymbol{\gamma}}$ is related to the incident beam energy $\left(\boldsymbol{E}_{p}\right)$ by

$$
E_{\gamma}=\frac{M}{m+M} E_{p}+Q
$$

where the $Q$-value $(\boldsymbol{Q})$ is $600.5 \mathrm{keV}$ for this reaction and $\boldsymbol{m} \& \boldsymbol{M}$ are ${ }^{16} \mathrm{O}$ and ${ }^{1} \mathrm{H}$ masses respectively, one can derive the proton beam energy from the gamma ray energy and subsequently derive the GVM calibration factor from Eq.2. In this case we found $E_{p}=1650.0 \pm 3.1 \mathrm{keV}$, which leads to a GVM calibration factor of $1.0044 \pm 0.0019$, assuming $0.04 \mathrm{keV}$ as the estimated energy loss in the stripper $\left(E_{s t r}\right)$ and using the nominal extraction voltage $\left(V_{\text {ext }}\right)$ of $30.9 \mathrm{kV}$. The uncertainty on the GVM calibration factor is derived in the "Uncertainty Budget" section.

For the triple-alpha source method, the position $\left(\boldsymbol{C} \boldsymbol{h}_{\boldsymbol{i}}\right.$ in channel number) of the $\mathrm{Si}$ and $\mathrm{O}$ edges as well as the position of the alpha peaks produced by the ${ }^{239} \mathrm{Pu},{ }^{241} \mathrm{Am} \&$ ${ }^{244} \mathrm{Cm}$ decays are carefully determined and the following set of equations is written for each detector and radio-isotope: 


$$
\begin{array}{ll}
k_{S i} E_{\alpha}-\Delta E_{P H D}^{S i}=C h_{S i} a+b & \text { Eq.4 } \\
k_{O} E_{\alpha}-\Delta E_{P H D}^{O}=C h_{O} a+b & \text { Eq.5 } \\
E_{A m}-\Delta E_{P H D}^{A m}-\Delta E_{S}=C h_{A m} a+b & \text { Eq.6 }
\end{array}
$$

where:

- $\quad \boldsymbol{k}_{\boldsymbol{i}}$ is the kinematical factor for the element $i$

- $\quad \boldsymbol{E}_{\boldsymbol{\alpha}}$ is the incident beam energy;

- $\quad \Delta \boldsymbol{E}_{\boldsymbol{P} \boldsymbol{H} \boldsymbol{D}}^{\boldsymbol{i}}$ represents the energy losses due to the detector entrance electrode $\&$ the dead layer, and the non-ionising (nuclear) processes for the particles backscattered by the element $i$ (or emitted by the radioactive element $i$ );

- $\quad \boldsymbol{C} \boldsymbol{h}_{\boldsymbol{i}}$ is the channel at which the particles backscattered by the element $i$ (or emitted by the radionuclide $i$ ) are detected;

- $\quad \boldsymbol{a} \& \boldsymbol{b}$ are the electronic gain \& offset of the pulse-height spectrometry system;

- $\quad \boldsymbol{E}_{\boldsymbol{A m}}$ is the energy of the most likely ${ }^{241} \mathrm{Am}$ decay (i.e. $5486 \mathrm{keV}$ ).

- $\Delta \boldsymbol{E}_{\boldsymbol{s}}$ is the energy loss in the sealing layer of the triple-alpha source (this factor takes account of the effective source thickness).

Resolving this system of equations allows one to determine the incident beam energy as well as the electronic gain and offset of the pulse-height spectrometry system. In this work we used two independent detectors and three different radio-isotopes, making 6 independent evaluations of the incident beam energy. The average of these measurements is found to be $2998.8 \pm 1.8 \mathrm{keV}$ where the uncertainty is given as a standard error on the mean at $99 \%$ of confidence (SE99, Eq.1). Since the RBS spectra were acquired for a terminal voltage $\left(V_{t}\right)$ set at $1478.0 \mathrm{kV}$, the GVM calibration factor derived from this measurement (through Eq.2) is $1.0079 \pm 0.0008$, assuming $0.47 \mathrm{keV}$ as the estimated energy loss in the stripper $\left(E_{s t r}\right)$ and using the nominal extraction voltage $\left(V_{\text {ext }}\right)$ of $20.0 \mathrm{kV}$. The uncertainty on the GVM calibration factor is derived in the "Uncertainty Budget" section.

Since the GVM calibration factor is routinely determined at the Surrey lon Beam Centre as a part of our quality assurance program [12,27], the long term stability of the GVM may also be assessed. Figure 4 shows 6 independent measurements of the GVM calibration factor performed after January 2014. Figure 5 shows similar results obtained between June 2012 and October 2013 (the GVM mechanism having been refurbished between these measurements). Table 3 summarises the results shown in figures $5 \& 6$. 


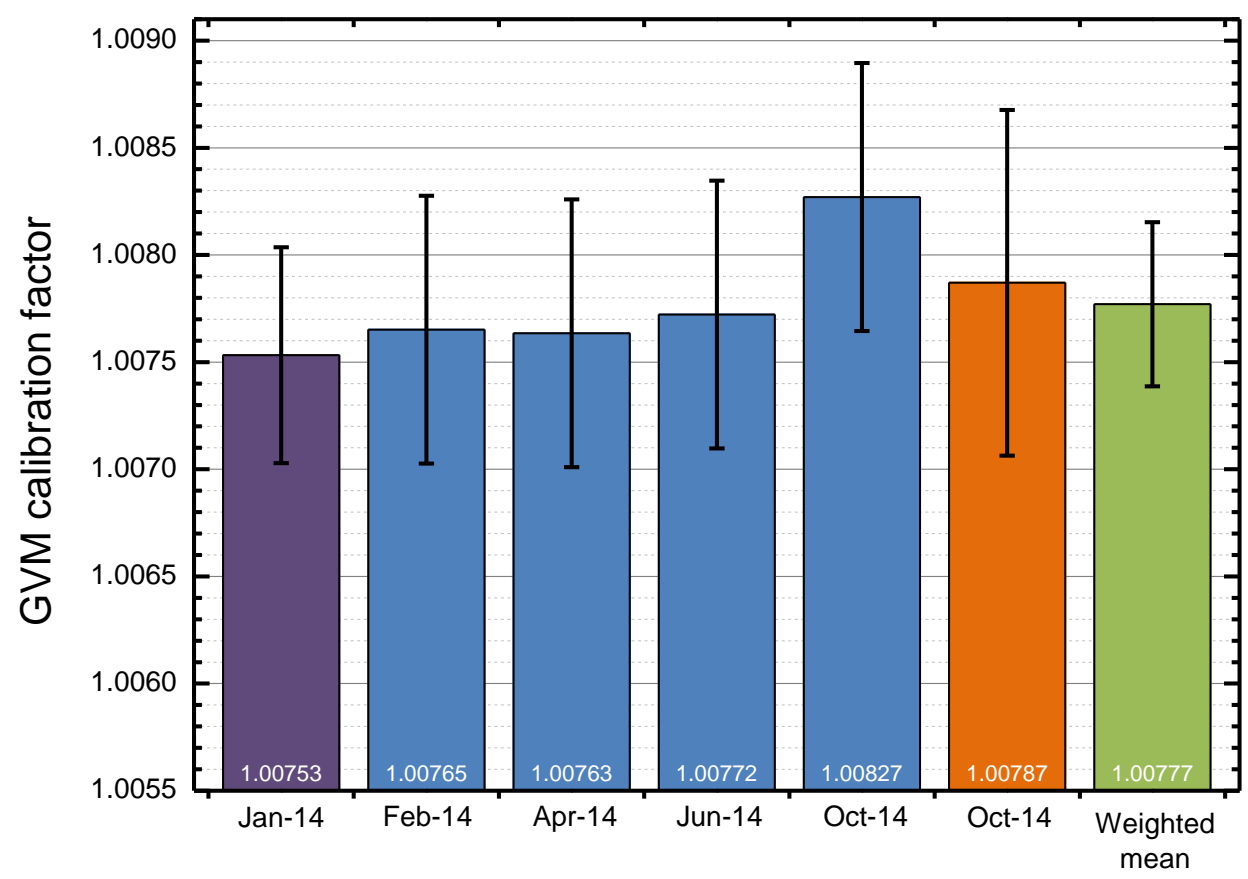

Figure 4: Time series for GVM calibration factor in 2014 (Surrey). These use the calibration methods ${ }^{16} \mathrm{O}(\alpha, \alpha){ }^{16} \mathrm{O}$ (in blue), ${ }^{27} \mathrm{Al}(\mathrm{p}, \gamma)^{28} \mathrm{Si}$ (in purple) or triple-alpha source (in orange). The error bars are given as the standard uncertainty associated with each calibration method (see "Uncertainty Budget" section). The weighted mean (in green) is calculated using $W_{i}=1 / U_{i}$ and its error bars are given as SE99 (Eq.1): $\mathbf{1 . 0 0 7 8} \pm \mathbf{0 . 0 0 0 4}$.

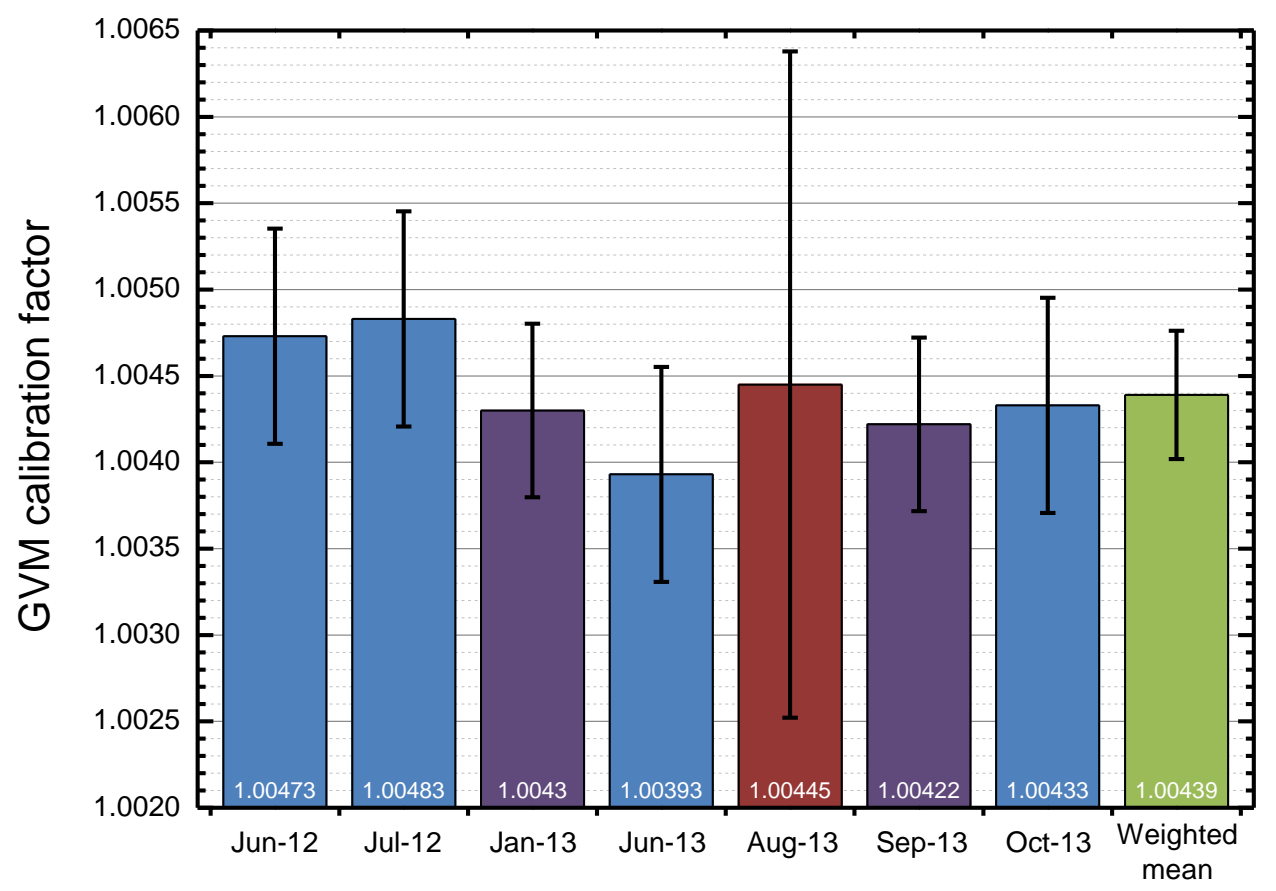

Figure 5: Time series for GVM calibration factor before 2014 (Surrey). These use the calibration methods ${ }^{16} \mathrm{O}(\alpha, \alpha){ }^{16} \mathrm{O}$ (in blue), ${ }^{16} \mathrm{O}(\mathrm{p}, \gamma)^{17} \mathrm{~F}$ (in red) or ${ }^{27} \mathrm{Al}(\mathrm{p}, \gamma)^{28} \mathrm{Si}$ (in purple). The error bars are given according to the standard uncertainty associated with each calibration method (see "Uncertainty Budget" section). The weighted mean (in green) is calculated using $W_{i}=1 / U_{i}$ and its error bars are given as SE99 (Eq.1): $\mathbf{1 . 0 0 4 4} \pm \mathbf{0 . 0 0 0 4}$. 


\begin{tabular}{|c|c|c|c|}
\hline \multirow{2}{*}{ Period } & Calibration method & \multicolumn{2}{|c|}{ GVM calibration factor } \\
\cline { 2 - 4 } & ${ }^{16} \mathrm{O}(\alpha, \alpha)^{16} \mathrm{O}$ & Value & Uncertainty (\%) \\
\hline \multirow{2}{*}{$\begin{array}{c}\text { June } 12- \\
\text { October } 13\end{array}$} & ${ }^{27} \mathrm{Al}(\mathrm{p}, \mathrm{\gamma})^{28} \mathrm{Si}$ & 1.00446 & $0.06 \%$ \\
\cline { 2 - 4 } & ${ }^{16} \mathrm{O}(\mathrm{p}, \mathrm{\gamma})^{17} \mathrm{~F}$ & 1.00426 & $0.05 \%$ \\
\hline \multirow{3}{*}{ January $14-$} & ${ }^{16} \mathrm{O}(\alpha, \alpha)^{16} \mathrm{O}$ & 1.00445 & $0.19 \%$ \\
\cline { 2 - 4 }$\ldots$ & ${ }^{27} \mathrm{Al}(\mathrm{p}, \mathrm{\gamma})^{28} \mathrm{Si}$ & 1.00782 & $0.06 \%$ \\
\cline { 2 - 4 } & Triple-alpha source & 1.00753 & $0.05 \%$ \\
\hline
\end{tabular}

Table 3: Summary of GVM calibration factors. These are derived from different calibration methods in two different time periods (between which the GVM has been refurbished). When repeated measurements are available, the mean value is reported in this table. The uncertainties are determined according to the uncertainty budgets given below.

\section{University of Namur (LARN)}

The GVM calibration factor of the Tandem accelerator installed at the University of Namur was determined using the ${ }^{16} \mathrm{O}(\alpha, \alpha){ }^{16} \mathrm{O}$ calibration method described in this work. In this case, the thickness of the dead layer was nevertheless directly measured using a triplealpha source for each detector using the method described by Lennard et al [8]. These thicknesses were found to be (305, 542 and 305) TFU of Si for (DetC, DetD and DetE), respectively.

The incident energy of the alpha beam was then set at $3.000 \mathrm{MeV}$, and spectra were obtained from $\mathrm{Ta}_{2} \mathrm{O}_{5} / \mathrm{C}$ and $\mathrm{SiO}_{2} / \mathrm{Si}$ samples along with the radioactive triple-alpha source positioned slightly below the scattering plane. The position (channel number) of the $\mathrm{Si}, \mathrm{Ta} \& \mathrm{O}$ edges as well as the position of the peaks produced by the ${ }^{239} \mathrm{Pu},{ }^{241} \mathrm{Am} \mathrm{\&}{ }^{244} \mathrm{Cm}$ decays were carefully determined and the system of equations described above (Eqs.4-6) was written for each radionuclide, each detector (i.e. three in this experiment) and each sample (i.e. $\mathrm{Ta}_{2} \mathrm{O}_{5} \& \mathrm{SiO}_{2}$ ), leading to 18 independent evaluations of the incident beam energy. The average of these measurements is found to be $2999.3 \pm 1.2 \mathrm{keV}$ where the uncertainty is given as the SE on the mean at $99 \%$ of confidence (SE99, Eq.1). This value should be compared to the indicated beam energy (given the calibration) of $3000.0 \mathrm{keV}$. 


\section{Uncertainty Budget}

The GVM calibration factor " $\boldsymbol{a}$ " is determined using Eq.2, and the uncertainty associated with this equation is given by:

$$
\frac{\Delta a}{a}=\sqrt{\left(\frac{\Delta E}{E}\right)^{2}+\left(\frac{\Delta V_{t}}{V_{t}}\right)^{2}}
$$

where $E=E_{p}-e V_{e x t}+E_{s t r}$ and $\Delta E=\sqrt{\Delta E_{p}^{2}+\left(e \Delta V_{e x t}\right)^{2}+\Delta E_{s t r}{ }^{2}}$.

The evaluation of each term $\left(\Delta V_{t}, \Delta V_{\text {ext }}, \Delta E_{s t r}, \Delta E_{p}\right)$ is discussed in turn for the four different experiments (resonant EBS, resonant and non-resonant NRA, and the triple-alpha source), with these terms and the resulting values of $\Delta a / a$ (Eq.7) summarised in Table 6.

According the High Voltage Engineering Europa (HVEE) specifications of the Tandetron $^{\mathrm{TM}}$, the terminal voltage stability is $\pm 200 \mathrm{~V}$ and the terminal voltage ripple is $\pm 25 \mathrm{~V}$ (using the anti-ripple phase-locked feedback from the capacitive pickup). These topdown consideration therefore enable an estimate of the total voltage setting precision: $\Delta V_{t}=200 \mathrm{~V}$ since ripple does not affect the average voltage.

According to the datasheet, the uncertainty associated with the Glassman extraction power supply for the ion source is dominated by the setting accuracy: $\Delta \mathrm{V}_{\mathrm{ext}}=280 \mathrm{~V}$.

The uncertainty associated with the energy loss in the stripper gas $\Delta \mathrm{E}_{\mathrm{Str}}$ is as hard to estimate as the energy loss $E_{\text {str }}$ itself. From a literature estimate of the normal stripper gas pressure (typically 1-5 mTorr for our accelerator [31]) we estimate that the energy loss for $3 \mathrm{MeV}{ }^{4} \mathrm{He}$ in the stripper (680 mm in length; $8 \mathrm{~mm}$ in diameter) is $470 \mathrm{eV}$, and guess an uncertainty of $100 \%$ of this. Similarly, the energy loss for $1 \mathrm{MeV}^{1} \mathrm{H}\left({ }^{27} \mathrm{Al}(\mathrm{p}, \gamma)^{28} \mathrm{Si}\right.$ method) is estimated at $70 \mathrm{eV}$ and that for $1.6 \mathrm{MeV}{ }^{1} \mathrm{H}\left({ }^{16} \mathrm{O}(\mathrm{p}, \gamma)\right)^{17} \mathrm{~F}$ method) is estimated at $45 \mathrm{eV}$.

\section{$\underline{16} 0(\alpha, \alpha)^{16} 0$ calibration method}

For a given scattering angle, the incident beam energy is directly derived from the shape and intensity of the ${ }^{16} \mathrm{O}$ peak. In this work, the scattering angle is determined with an accuracy of 0.2 degrees. Such small scattering angle variation does not significantly affect the peak shape in the cross-section function, but it does vary the peak intensity by about $2 \%(0.3 \%)$ for the scattering angle of $150^{\circ}\left(170^{\circ}\right)$, which translates to an energy variation of about $0.5 \mathrm{keV}(<0.1 \mathrm{keV})$ for the given scattering angle. It should be highlighted that the ${ }^{16} \mathrm{O}$ peak shape strongly constrains the beam energy during the fitting procedure: that is, the ${ }^{16} \mathrm{O}$ peak shape given by the incident energy and the electronic gain is accordingly adjusted (given the PHD) for minimising $\chi^{2}$. The beam energy determination is therefore strongly related to the cross-section function itself: a study of the literature 
shows large discrepancies of the resonance position (Table 4) leading to a SE99 (Eq.1) on the weighted mean of $3.1 \mathrm{keV}$. However, we will show that a comparison of the ${ }^{16} \mathrm{O}(\alpha, \alpha)^{16} \mathrm{O}$ method to other calibration methods allows one to reduce the uncertainty on the ${ }^{16} \mathrm{O}(\alpha, \alpha)^{16} \mathrm{O}$ resonance energy to $1.0 \mathrm{keV}$ (see Table 7 and its discussion below).

\begin{tabular}{|c|c|c|}
\hline References & Position of the resonance (keV) & Uncertainty (keV) \\
\hline J.R. Cameron [32] & 3045.0 & 10.0 \\
\hline J.D. MacArthur et al. [33] & 3035.9 & 2.5 \\
\hline Z.L. Wang et al. [34] & 3042.0 & 3.2 \\
\hline R.A. Jarjis [35] & 3042.0 & 3.9 \\
\hline J.A. Leavitt et al. [36] & 3034.0 & 4.0 \\
\hline H.S. Cheng et al. [37] & 3035.0 & 6.0 \\
\hline V.I. Soroka et al. [38] & 3039.0 & 5.0 \\
\hline J. Demarche \& G. Terwagne [39] & 3037.4 & 1.9 \\
\hline Weighted mean & 3038.2 & \\
\hline SD & 3.1 & \\
\hline SE99 (Eq.1) & 3.1 & \\
\cline { 1 - 2 } & &
\end{tabular}

Table 4: Literature evaluation of the position (maximum value) of the ${ }^{16} \mathrm{O}(\alpha, \alpha){ }^{16} \mathrm{O}$ resonance at $3038.2 \pm 3.1 \mathrm{keV}$. The weighted mean is calculated using $w_{i}=1 / U_{i}$. It should be noted that the results of Demarche \& Terwagne have been re-evaluated as shown in the Annexe.

Considering the uncertainties on the scattering angle, the resonance energy, and the peak position determination [25], the uncertainty on the incident beam energy determination is about $1.2 \mathrm{keV}$. The uncertainty derived for $\Delta a / a$ from Eq.7 is therefore $0.05 \%$ (see Table 6) when considering $E_{p}=3065 \mathrm{keV}$, the mean energy of the dataset used for the GVM calibration procedure. However, as discussed above, the spectra acquired around $3.0 \mathrm{MeV}$ are rather insensitive to the PHD of the detector which cannot be determined at better than $20 \%$, translating into a GVM calibration factor variation of about $0.04 \%$. The combined uncertainty applying to this ${ }^{16} \mathrm{O}(\alpha, \alpha){ }^{16} \mathrm{O}$ calibration method is therefore estimated as $\mathbf{0 . 0 6 \%}$.

Regarding the electronic gain derived from the dataset collected around $3 \mathrm{MeV}$, the accuracy is limited by the lack of sensitivity to the PHD of the detector. One can demonstrate with a covariance analysis that the PHD cannot be determined with accuracy better than $20 \%$ which translates to an uncertainty of about $0.4 \%$ for the electronic gain determination. But a second set of data acquired at $1.5 \mathrm{MeV}$ on the same standard calibration sample helps reducing the uncertainty on the electronic gain down to about $0.1 \%$, as discussed elsewhere [9]. 


\section{$\underline{\text { Resonant }{ }^{27} \text { Al }(p, \gamma){ }^{28} \text { Si calibration method }}$}

In this case the parameter $E_{p}$ should be read as the energy of the resonance (991.90 $\pm 0.04 \mathrm{keV}[1])$ and the experimental terminal voltage $\left(V_{t}=479.92 \pm 0.01 \mathrm{kV}\right)$ is derived from the Boltzmann curve fitting (Figure 3). The energy loss in the stripper channel $\left(E_{s t r}\right)$ should also be adapted for matching a proton beam around $1 \mathrm{MeV}\left(E_{s t r} \cong 0.07 \mathrm{keV}\right.$ in this case) while the terminal voltage stability and the uncertainty on the extraction voltage are exactly the same since we are using the same facility for producing both alpha or proton beams.

\section{Non-resonant ${ }^{16} \mathrm{O}(p, \gamma)^{17} \mathrm{~F}$ calibration method}

For this third method the incident beam energy $\left(E_{p}\right)$ is derived from Eq.3. The uncertainty applying to $E_{p}$ ought to take into account the uncertainties on the mass excesses of the nuclei involved in this direct capture reaction. However, this contribution is negligible compared to the uncertainty derived from the HPGe calibration. In this work the HPGe detector was calibrated using only 4 lines (see Experimental details) and the spectra were recorded on only 2048 channels, leading to a rather poor electronic gain determination (uncertainty was about $0.18 \%$ ) and thus a rather poor incident beam energy determination $\left(E_{p}=1650.0 \pm 3.1 \mathrm{keV}\right)$. The contribution of the other terms derived from Eq. 2 are very similar to the other calibration methods (see Table 6). Clearly, the attainable accuracy of this method would be greatly improved with more attention to the electronic gain determination of the HPGe detector.

\section{Triple-alpha source calibration method}

For this last method the incident beam energy is derived from the system of equations Eq. 4-6. The result obtained is affected by the various contributions summarised in Table 5. It should be noted that the uncertainty on the PHD determination given in this Table is only $5 \%$ while we said $20 \%$ above. This is because, for the triple-alpha source measurements, we used the full calibration method described elsewhere [9] which is capable of determining the PHD at 5\%. Regarding the measurements performed at Namur, the dead layer of each detector was directly measured using the triple-alpha source. In this case, the precision of the PHD determination is limited by the accuracy at which the alphapeaks positions can be determined: namely, 0.25 channel. Knowing the electronic gain (i.e. about $3.4 \mathrm{keV} / \mathrm{channel}$ for all of the three detectors) and the Si stopping power for the alpha particles around $5.5 \mathrm{MeV}$ (i.e. mean energy of the triple-alpha source), that translates to an uncertainty of about $9 \%$ on the PHD determination. But of course each peak of the triple-alpha source can be separately used for assessing the dead layer thickness, reducing the uncertainty to about $5 \%$. 
Taking into account all of the contributions given in Table $\mathbf{5}$ as well as the SE99 on the mean (Eq.1: $1.8 \mathrm{keV}$ for the measurements performed at Surrey and $1.2 \mathrm{keV}$ for Namur), the uncertainty on the incident beam energy determination is about $2.3 \mathrm{keV}$ for the measurements performed at Surrey and $1.9 \mathrm{keV}$ for Namur. The contribution of the other terms derived from Eq. 2 are very similar to the other calibration methods (see Table 6).

\begin{tabular}{|l|c|c|}
\hline & Uncertainty on the parameter & $\begin{array}{c}\text { Variation of the derived incident } \\
\text { beam energy (keV) }\end{array}$ \\
\hline Scattering angle & $\pm 0.5^{\circ}$ (around $\left.150^{\circ}\right)$ & 0.3 \\
\hline PHD & $\pm 5 \%$ & 0.5 \\
\hline Signal position & \pm 0.25 channel & 1.2 \\
\hline Si stopping power & $\pm 2 \%$ & 0.4 \\
\hline Energy loss in sealing source layer & $\pm 0.5 \mathrm{keV}$ & 0.3 \\
\hline
\end{tabular}

Table 5: Beam energy uncertainty from the triple-alpha source method. Values reported in this table were determined by trial-and-error.

\section{Summary for GVM factor uncertainties}

Table 6 summarises the uncertainties of the four methods of determining the GVM calibration factor that we have explored here. Clearly, if the gamma spectrometer could be calibrated more accurately for the non-resonant ${ }^{16} \mathrm{O}(\mathrm{p}, \gamma)^{17} \mathrm{~F}$ reaction, the uncertainties of this method could be reduced.

\begin{tabular}{|c|c|c|c|c|c|c|c|}
\hline Beam & $\begin{array}{c}E_{p} \\
\mathrm{keV}\end{array}$ & $\begin{array}{c}\Delta V_{t} \\
\mathrm{kV}\end{array}$ & $\begin{array}{c}\Delta V_{\text {ext }} \\
\mathrm{kV}\end{array}$ & $\begin{array}{c}\Delta E_{\text {str }} \\
\mathrm{keV}\end{array}$ & $\begin{array}{c}\Delta E_{p} \\
\mathrm{keV}\end{array}$ & $\begin{array}{c}\Delta a / a \\
\%\end{array}$ & Comment \\
\hline${ }^{4} \mathrm{He}$ & 3065 & 0.2 & 0.28 & 0.47 & 1.2 & $0.05 \%$ & ${ }^{16} \mathrm{O}(\alpha, \alpha)^{16} \mathrm{O}$ (resonant) \\
\hline${ }^{1} \mathrm{H}$ & 992 & 0.2 & 0.28 & 0.07 & 0.04 & $0.05 \%$ & ${ }^{27} \mathrm{Al}(\mathrm{p}, \gamma)^{28} \mathrm{Si}$ (resonant) \\
\hline${ }^{1} \mathrm{H}$ & 1650 & 0.2 & 0.28 & 0.04 & 3.1 & $0.19 \%$ & ${ }^{16} \mathrm{O}(\mathrm{p}, \gamma)^{17} \mathrm{~F}$ (non-resonant) \\
\hline${ }^{4} \mathrm{He}$ & 3000 & 0.2 & 0.28 & 0.47 & 2.3 & $0.08 \%$ & Triple-alpha, ${ }^{239} \mathrm{Pu}^{241} \mathrm{Am}^{244} \mathrm{Cm}$ at Surrey \\
\hline${ }^{4} \mathrm{He}$ & 3000 & 0.2 & 0.28 & 0.47 & 1.9 & $0.07 \%$ & Triple-alpha, ${ }^{239} \mathrm{Pu}^{241} \mathrm{Am}^{244} \mathrm{Cm}$ at Namur \\
\hline
\end{tabular}

Table 6: Uncertainties of the various methods of determining beam energy, from Eq.7 


\section{Discussion}

\section{Beam energy calibration}

The final results obtained for the GVM calibration are summarised in Table 2. For each nominal terminal voltage, the incident beam energy is derived from the fitting procedure for each detector. The mean $\frac{E_{\text {DetA }}}{E_{\text {Det } B}}$ ratio $\left(5^{\text {th }}\right.$ column) is very close to unity with an uncertainty (SE99, see Eq.1) of only $0.005 \%$, which demonstrates how sensitive the ${ }^{16} \mathrm{O}$ peak shape is to the incident beam energy.

It should be noted that the value of the PHD found in this fitting procedure depends only weakly on beam energy. This is because the energy lost in the dead layer, and the non-ionising energy loss as a proportion of the incident energy, are both reduced for higher backscattered particle energies. Of course, when the PHD value is changed, the electronic gain also needs adjusting in the fitting procedure, so that for a large PHD variation the incident energy is also modified. But in this case neither the Au-Ni-Si edges nor the ${ }^{16} \mathrm{O}$ peak shape are correctly fitted and $\chi^{2}$ is significantly higher. One can demonstrate (by trial and error) that a variation of $20 \%$ of the PHD value affects the GVM calibration factor only by about $0.04 \%$, where $\chi^{2}$ is increased by about $0.6 \%$ which we take as the smallest detectable change. Our beam energy calibration method is therefore rather insensitive to the PHD.

For each nominal terminal voltage, the average of the energies derived from both detectors is used for calculating the GVM calibration factor through Eq.2. In this experiment (Jun-14 in Figure 4), the mean value of the GVM calibration factor is equal to 1.00772. The uncertainty of the mean (SE99, Eq.1) gives the precision of the calibration method: $0.004 \%$ in this work, but usually around $\mathbf{0 . 0 1 \%}$ (that is, $300 \mathrm{eV}$ in $3 \mathrm{MeV}$ ).

Figures $5 \& 6$ show that the ${ }^{16} \mathrm{O}(\alpha, \alpha)^{16} \mathrm{O}$ calibration method described in this work has been validated by comparison to three other independent calibration methods (using the triple-alpha source, and the resonant and non-resonant capture reactions), since all the GVM calibration factors measured in this work are in agreement with each other within the uncertainties. It should also be noted that the standard deviation of the measurements performed following this ${ }^{16} \mathrm{O}(\alpha, \alpha){ }^{16} \mathrm{O}$ calibration method is only $0.033 \%$, suggesting a reproducibility of about $1 \mathrm{keV}$ in $3 \mathrm{MeV}$.

Moreover, the set of GVM calibration factor measurements shown in this work can be used for further reducing the uncertainty on the resonance energy. Indeed, if the ${ }^{16} \mathrm{O}(\alpha, \alpha){ }^{16} \mathrm{O}$ resonance energy of the $3038.2 \mathrm{keV}$ reaction is correct, then the ratio between the GVM calibration factors derived from this and the other calibration methods should be unity. The results are summarised in Table 7 . The weighted mean of these ratios is found 
to be $1.00016 \pm 0.00033$ (SE99 uncertainty, see Eq.1). Since this is indistinguishable from unity we conclude that any error on the ${ }^{16} \mathrm{O}(\alpha, \alpha){ }^{16} \mathrm{O}$ resonance energy must be smaller than the uncertainty on the mean GVM calibration factor ratio: that is, the resonance energy is $3038.2 \pm 1.0 \mathrm{keV}$. The accuracy (combined standard uncertainty) of the ${ }^{16} \mathrm{O}(\alpha, \alpha){ }^{16} \mathrm{O}$ calibration method described in this work is therefore evaluated at $\mathbf{0 . 0 6 \%}$.

\begin{tabular}{|c|c|c|c|c|}
\hline & Period & Calibration methods used & $\begin{array}{l}\text { GVM calibration factor } \\
\text { ratio }\end{array}$ & Uncertainty \\
\hline \multirow{4}{*}{ Surrey } & \multirow{2}{*}{$\begin{array}{l}\text { June } 12- \\
\text { October } 13\end{array}$} & ${ }^{16} \mathrm{O}(\alpha, \alpha){ }^{16} \mathrm{O} /{ }^{27} \mathrm{Al}(p, \gamma){ }^{28} \mathrm{Si}$ & 1.000197 & $0.07 \%$ \\
\hline & & ${ }^{16} \mathrm{O}(\alpha, \alpha){ }^{16} \mathrm{O} /{ }^{16} \mathrm{O}(p, \gamma){ }^{17} \mathrm{~F}$ & 1.000285 & $0.07 \%$ \\
\hline & \multirow{2}{*}{$\begin{array}{c}\text { January } 14- \\
\ldots\end{array}$} & ${ }^{16} \mathrm{O}(\alpha, \alpha){ }^{16} \mathrm{O} /{ }^{27} \mathrm{Al}(p, \gamma){ }^{28} \mathrm{Si}$ & 1.000005 & $0.20 \%$ \\
\hline & & ${ }^{16} \mathrm{O}(\alpha, \alpha){ }^{16} \mathrm{O} /$ Triple-alpha source & 0.999950 & $0.09 \%$ \\
\hline \multirow[t]{4}{*}{ Namur } & \multirow[t]{4}{*}{ Apr-14 } & ${ }^{16} \mathrm{O}(\alpha, \alpha){ }^{16} \mathrm{O} /$ Triple-alpha source & 1.000243 & $0.08 \%$ \\
\hline & & Weighted mean & 1.000165 & \\
\hline & & SD & $0.015 \%$ & \\
\hline & & SE99 (Eq.1) & $0.033 \%$ & \\
\hline
\end{tabular}

Table 7: Comparison of the ${ }^{16} \mathrm{O}(\alpha, \alpha)^{16} \mathrm{O}$ with other calibration methods. The fourth column of this table shows the ratio of the GVM calibration factor determined with our ${ }^{16} \mathrm{O}(\alpha, \alpha)^{16} \mathrm{O}$ and the other calibration methods. The uncertainty on the ratio is obtained by adding the uncertainty of each calibration method in quadrature. That is, $0.05 \%$ for the ${ }^{27} \mathrm{Al}(\mathrm{p}, \gamma)^{28} \mathrm{Si}$; $0.19 \%$ for the ${ }^{16} \mathrm{O}(\mathrm{p}, \gamma){ }^{17} \mathrm{~F} ; 0.08 \%$ or $0.07 \%$ for the Triple-alpha source and $0.05 \%$ for the ${ }^{16} \mathrm{O}(\alpha, \alpha){ }^{16} \mathrm{O}$ calibration method (see Table 6). The latter is obtained considering that the $3038.2 \mathrm{keV}$ resonance energy is exact (i.e. $\Delta E_{p}=0 \mathrm{keV}$ in Eq.7).

Finally, it is worth mentioning that the GVM stability of the Tandem accelerator is remarkable. Figure 3 shows that the GVM is very stable (and reproducible) for a time period of hours, but Figures $5 \& 6$ emphasise this stability for much longer period. The significant difference between the mean GVM calibration factors is due to a refurbishment in early January 2014.

\section{Electronic gain calibration}

The electronic gain of the pulse-height spectrometry system is simultaneously determined with the beam energy when processing the dataset acquired around 3.0 MeV. The SE99 (Eq.1) on the mean electronic gain (Table 1) demonstrates the very high precision of this technique (about $\mathbf{0 . 0 3 \%}$ for both detectors). However, as discussed above, the spectra acquired at 3.0 MeV on the calibration sample are rather insensitive to the PHD value: the dataset can be equivalently fitted (i.e. similar $\chi^{2}$ values) with PHD values varied by $20 \%$, but such a PHD variation leads to an electronic gain variation of about $0.2 \%$. The accuracy of the electronic gain determination achieved in this work is therefore estimated $\mathbf{0 . 4 \%}$, which is already very satisfying but unfortunately not yet quite good enough for high accuracy RBS measurements $[12,27]$. 


\section{Elastic (non-Rutherford) backscattering (EBS) cross-sections}

This method depends on the use of an evaluated EBS cross-section function for the ${ }^{16} \mathrm{O}(\alpha, \alpha)^{16} \mathrm{O}$ reaction [5], that is, a nuclear scattering model using R-matrix methods and potential calculations to calculate the scattering cross-section [40]. Although these EBS cross-section functions vary extremely rapidly with energy at resonances, their shapes are strongly constrained by rather simple considerations. This is a well-known resonance at $3038.2 \pm 2.0 \mathrm{keV}$ (giving a ${ }^{20} \mathrm{Ne}^{*}$ excitation energy of about $7156 \mathrm{keV}$ ), a width of $\Gamma=8.1 \pm 0.3 \mathrm{keV}$, and a spin and parity of $\mathrm{J}^{\pi}=3^{-}$(Table 20.22 in Tilley et al [41]). Note that spin and parity are unambiguously assigned from a number of different nuclear methods (including EBS cross-sections as a function of angle). In this case the resonance can be modelled entirely by potential scattering from the second member of the $\mathrm{K}^{\pi}=0^{-}$band in cluster models of the ${ }^{20} \mathrm{Ne}$ nucleus $[5,33,42]$.

To estimate the uncertainty introduced by using evaluated cross-section functions to obtain beam energies from fits to backscattering spectra we do not have to estimate the uncertainty of the EBS cross-sections, which is hard to do in general with attempts so far having been unsatisfactory: Mayer [43] compares datasets statistically with interesting results. But Gai \& Gurbich [44] point out that Mayer's method is invalid in principle, instead constructing the covariance matrix: however, their calculated uncertainties near resonances are certainly overestimated.

For the specific case of the $3038 \mathrm{keV}$ resonance, the width is quite well-known with good measurements of the resonance shape: the main uncertainty is in the position, which is being freely fitted. Our value of $3038.2 \mathrm{keV}$ happens to coincide exactly with the data compilation made by Tilley et al [41], but with a significantly smaller uncertainty (1.0 keV rather than $2.0 \mathrm{keV}$ ).

\section{Conclusion}

We have presented an original method using the ${ }^{16} \mathrm{O}(\alpha, \alpha)^{16} \mathrm{O}$ resonance to calibrate the beam energy and the chain of detection at the same time. The accuracy of this method is mainly limited by the accuracy at which the energy of the resonance is known. We have re-evaluated the energy of this resonance to be $3038.2 \pm 3.1 \mathrm{keV}$ from a review of the literature (see Table 4). However, this resonance energy is entirely consistent with repeated measurements using three other independent calibration methods as shown in Table 7. Together, these show that the uncertainty of the resonance energy is not larger than $1.0 \mathrm{keV}(0.03 \%)$. The traceable combined uncertainty of this calibration was therefore demonstrated to be $0.06 \%$ for the beam energy ( $1.8 \mathrm{keV}$ at $3 \mathrm{MeV})$. 
By this method the electronic gain is determined at about $0.4 \%$, but this parameter is better determined at lower energies, as we describe elsewhere [9].

This calibration method is relatively fast $(\sim 0.5 \mathrm{~h}$ for the offset measurement; $\sim 0.5 \mathrm{~h}$ for the acquisition of the dataset around $3 \mathrm{MeV} ; \sim 1 \mathrm{~h}$ for the data processing), and is suitable for any kind of accelerator system, although we have implemented it on systems depending on very reliable terminal voltage stabilisation.

\section{Acknowledgements}

We are grateful to A.F.Gurbich for extensive discussions, and for his adjusting the parameters of SigmaCalc for the ${ }^{16} \mathrm{O}(\alpha, \alpha)^{16} \mathrm{O}$ reaction to give a maximum at $3038 \mathrm{keV}$.

\section{Annexe}

The measurement of the ${ }^{16} \mathrm{O}(\alpha, \alpha)^{16} \mathrm{O}$ cross-section function performed by Demarche \& Terwagne [39] was carried out with a 2 MV Tandem accelerator from HVEE. The GVM calibration was performed using variety of $(p, n)$ neutron threshold and nuclear resonant reactions induced by proton beam (Table 8 ). The relationship between the theoretical terminal voltage (that is, the terminal voltage at which the threshold/resonance is supposed to occur: $V_{t}$ ) and the actual terminal voltage (that is, the measurement: $V_{\text {expt }}$ ) found by Demarche \& Terwagne is:

$$
V_{t}=V_{\text {expt }} \times 1.0065+33.0 \quad \text { Eq.A1 }
$$

Using that GVM calibration factor (1.0065) they derived the position of the ${ }^{16} \mathrm{O}(\alpha, \alpha){ }^{16} \mathrm{O}$ resonance at

$$
E_{R}=(3031.7 \pm 0.4) \mathrm{keV} \quad \text { Eq.A2 }
$$

However, performing the linear regression of the data shown in Table 8 we found:

$$
V_{t}=V_{\text {expt }} \times 1.0087+16.06 \quad \text { Eq.A3 }
$$

which is significantly different from the GVM calibration factor used in [39] for determining the position of the resonance. It turned out that Demarche \& Terwagne used a wrong GVM calibration factor (1.0065) measured for the same accelerator at another occasion. Using the GVM calibration factor determined above (Eq.A3), one can re-evaluate the position of the resonance:

$$
E_{R}^{\text {corrected }}=(3031.7-33) \times \frac{1.0087}{1.0065}+32.12=3037.4 \mathrm{keV} \quad \text { Eq.A4 }
$$

since the ${ }^{16} \mathrm{O}(\alpha, \alpha)^{16} \mathrm{O}$ cross-section function around $3 \mathrm{MeV}$ was measured with a ${ }^{4} \mathrm{He}^{+}$ beam. In order to evaluate the uncertainty of this corrected value, we have recalculated the theoretical voltage of each threshold/resonance when considering a GVM calibration 
factor of 1.0087 and an injection energy of $32.12 \mathrm{keV}$ (Eq.A3), and we have compared these values to the experimental ones (Table 89). The standard error (given at $99 \%$ of confidence) on the mean gives the uncertainty on the terminal voltage for this measurement. The ${ }^{16} \mathrm{O}(\alpha, \alpha)^{16} \mathrm{O}$ resonance energy is therefore estimated at $3037.4 \pm 1.9 \mathrm{keV}$.

TABLE II. Nuclear reaction thresholds and resonances used for calibrating the terminal voltage of the Tandetron accelerator. $V_{t}$ and $V_{\text {expt }}$ are, respectively, the theoretical and the experimental voltages of the terminal.

\begin{tabular}{|c|c|c|c|c|}
\hline Reaction & $\begin{array}{c}E_{\text {th }} \text { or } E_{R} \\
(\mathrm{MeV})\end{array}$ & $\begin{array}{c}V_{t} \\
(\mathrm{kV})\end{array}$ & $\begin{array}{l}V_{\text {expt }} \\
(\mathrm{kV})\end{array}$ & $\begin{array}{c}\text { Reaction } \\
\text { type }\end{array}$ \\
\hline${ }^{7} \mathrm{Li}(p, n)^{7} \mathrm{Be}$ & 1.88036 & 923.68 & 916.94 & Threshold \\
\hline${ }^{9} \mathrm{Be}(p, n){ }^{9} \mathrm{~B}$ & 2.05743 & 1012.215 & 1002.88 & Threshold \\
\hline${ }^{13} \mathrm{C}(p, n){ }^{13} \mathrm{~N}$ & 3.23557 & 1601.28 & 1587.96 & Threshold \\
\hline${ }^{19} \mathrm{~F}(p, \alpha \gamma){ }^{16} \mathrm{O}$ & 0.34046 & 153.73 & 152.42 & Resonance \\
\hline${ }^{19} \mathrm{~F}(p, \alpha \gamma){ }^{16} \mathrm{O}$ & 0.87211 & 419.555 & 416.58 & Resonance \\
\hline${ }^{15} \mathrm{~N}(p, \alpha \gamma){ }^{12} \mathrm{C}$ & 0.42900 & 198.00 & 197.00 & Resonance \\
\hline
\end{tabular}

Table 8: Table extracted from Demarche \& Terwagne [39] showing the neutron thresholds and nuclear resonant reactions induced by protons used for GVM calibration purposes.

\begin{tabular}{|c|c|c|c|c|}
\hline J. Demarche \& G. Terwagne [39] & $E(k e V)$ & $V_{t}$ & $V_{\text {expt }}$ & $V_{\text {expt }}-V_{t}(k V)$ \\
\hline${ }^{7} \mathrm{Li}(p, n)^{7} \mathrm{Be}$ & 1880.36 & 916.15 & 916.94 & 0.79 \\
\hline${ }^{9} \mathrm{Be}(p, n){ }^{9} \mathrm{~B}$ & 2057.24 & 1003.83 & 1002.88 & -0.95 \\
\hline${ }^{13} \mathrm{C}(p, n){ }^{13} \mathrm{~N}$ & 3235.48 & 1587.87 & 1587.96 & 0.09 \\
\hline${ }^{19} \mathrm{~F}(p, \alpha \gamma)^{16} \mathrm{O}$ & 340.46 & 152.84 & 152.42 & -0.42 \\
\hline${ }^{19} \mathrm{~F}(\mathrm{p}, \alpha \gamma){ }^{16} \mathrm{O}$ & 872.11 & 416.37 & 416.58 & 0.21 \\
\hline${ }^{15} \mathrm{~N}(p, \alpha \gamma){ }^{12} \mathrm{C}$ & 429.00 & 196.73 & 197.00 & 0.27 \\
\hline & & \multicolumn{2}{|c|}{ Average } & 0.00 \\
\hline & & \multicolumn{2}{|c|}{ SD } & 0.60 \\
\hline & & \multicolumn{2}{|c|}{ SE99 (Eq.1) } & 0.94 \\
\hline
\end{tabular}

Table 9: Comparison between the theoretical (determined when considering a GVM calibration factor of 1.0087 and an injection energy of $32.12 \mathrm{keV}$ ) and the experimental voltages observed for each threshold/resonance nuclear reaction. 


\section{References}

[1] J.B. Marion, Accelerator Calibration Energies, Rev Mod Phys, 38 (1966) 660-668.

[2] R.O. Bondelid, E.E.D. Whiting, $(p, n)$ Threshold-Curve Shapes and Measurements of Threshold Energy with 1H+ Beams, Phys Rev B, 134 (1964) B591-B594.

[3] C. Rolfs, W.S. Rodney, S. Durrance, H. Winkler, New Method for Measurement of Proton-Beam Energies, Nucl Phys A, A240 (1975) 221-234.

[4] J.D. Seagrave, J.E. Brolley, J.G. Beery, Absolute Velocity Gauge for Nuclear Particle Beams, Rev Sci Instrum, 35 (1964) 1290-\&.

[5] A.F. Gurbich, Evaluated differential cross-sections for IBA, Nucl Instrum Meth B, 268 (2010) 1703-1710.

[6] A.F. Gurbich, The SigmaCalc calculator, http://sigmacalc.iate.obninsk.ru/ .

[7] C.R. Nita, D. Pantelica, G. Cata-Danil, H. Petrascu, G. Velisa, P. Ionescu, D.G. Ghita, M. Filipescu, The Energy Calibration of the Bucharest Fn Tandem Accelerator, $U$ Politeh Buch Ser A, 74 (2012) 141-152.

[8] W.N. Lennard, S.Y. Tong, G.R. Massoumi, L. Wong, On the Calibration of Low-Energy Ion Accelerators, Nucl Instrum Meth B, 45 (1990) 281-284.

[9] J.L. Colaux, C. Jeynes, Accurate electronics calibration for particle backscattering spectrometry, Anal. Methods, (2015) http://dx.doi.org/10.1039/c4ay02988g

[10] C. Pascual-Izarra, N.P. Barradas, Introducing routine pulse height defect corrections in IBA, Nucl Instrum Meth B, 266 (2008) 1866-1870.

[11] C. Jeynes, N.P. Barradas, E. Szilágyi, Accurate Determination of Quantity of Material in Thin Films by Rutherford Backscattering Spectrometry, Anal Chem, 84 (2012) 60616069.

[12] J.L. Colaux, C. Jeynes, High accuracy traceable Rutherford backscattering spectrometry of ion implanted samples, Anal. Methods, 6 (2014) 120-129.

[13] A. Simon, C. Jeynes, R.P. Webb, R. Finnis, Z. Tabatabian, P.J. Sellin, M.B.H. Breese, D.F. Fellows, R. van den Broek, R.M. Gwilliam, The new Surrey ion beam analysis facility, Nucl Instrum Meth B, 219 (2004) 405-409.

[14] C. Jeynes, N.P. Barradas, M.J. Blewett, R.P. Webb, Improved ion beam analysis facilities at the University of Surrey, Nucl Instrum Meth B, 136 (1998) 1229-1234.

[15] N.P. Barradas, C. Jeynes, R.P. Webb, Simulated annealing analysis of Rutherford backscattering data, Appl Phys Lett, 71 (1997) 291-293.

[16] N.P. Barradas, C. Jeynes, Advanced physics and algorithms in the IBA DataFurnace, Nucl Instrum Meth B, 266 (2008) 1875-1879.

[17] H.H. Andersen, F. Besenbacher, P. Loftager, W. Moller, Large-Angle Scattering of Light-lons in the Weakly Screened Rutherford Region, Phys Rev A, 21 (1980) 18911901.

[18] J.F. Ziegler, Srim-2003, Nucl Instrum Meth B, 219 (2004) 1027-1036.

[19] J.F. Ziegler, M.D. Ziegler, J.P. Biersack, SRIM - The stopping and range of ions in matter (2010), Nucl Instrum Meth B, 268 (2010) 1818-1823.

[20] S.L. Molodtsov, A.F. Gurbich, Simulation of the pulse pile-up effect on the pulseheight spectrum, Nucl Instrum Meth B, 267 (2009) 3484-3487.

[21] N.P. Barradas, C. Jeynes, S.M. Jackson, RBS/simulated annealing analysis of buried SiCOx layers formed by implantation of O into cubic silicon carbide, Nucl Instrum Meth B, 136 (1998) 1168-1171. 
[22] W.N. Lennard, H. Xia, J.K. Kim, Revisiting the stopping powers of Si and SiO2 for He-4 ions: 0.5-2.0 MeV, Nucl Instrum Meth B, 215 (2004) 297-307.

[23] F. Yang-Wallentin, K.G. Joreskog, H. Luo, Confirmatory Factor Analysis of Ordinal Variables With Misspecified Models, Struct. Equ. Modeling, 17 (2010) 392-423.

[24] C. Jeynes, N.P. Barradas, P.K. Marriott, G. Boudreault, M. Jenkin, E. Wendler, R.P. Webb, Elemental thin film depth profiles by ion beam analysis using simulated annealing - a new tool, J Phys D Appl Phys, 36 (2003) R97-R126.

[25] A.C. Kimber, C. Jeynes, An Application of the Truncated 2-Piece Normal-Distribution to the Measurement of Depths of Arsenic Implants in Silicon, Appl. Stat. - J. Roy. Stats. Soc. C, 36 (1987) 352-357.

[26] W. Hosler, R. Darji, ON THE NONLINEARITY OF SILICON DETECTORS AND THE ENERGY CALIBRATION IN RBS, Nucl Instrum Meth B, 85 (1994) 602-606.

[27] J.L. Colaux, C. Jeynes, K.C. Heasman, R.M. Gwilliam, Certified ion implantation fluence by high accuracy RBS, Analyst, 2015, http://dx.doi.org/10.1039/C4AN02316A

[28] Z. Siketić, I.B. Radović, E. Alves, N.P. Barradas, Stopping power of 11B in Si and TiO2 measured with a bulk sample method and Bayesian inference data analysis, Nucl Instrum Meth B, 268 (2010) 1768-1771.

[29] N.P. Barradas, K. Arstila, G. Battistig, M. Bianconi, N. Dytlewski, C. Jeynes, E. Kotai, G. Lulli, M. Mayer, E. Rauhala, E. Szilágyi, M. Thompson, Summary of "IAEA intercomparison of IBA software", Nucl Instrum Meth B, 266 (2008) 1338-1342.

[30] N.P. Barradas, E. Alves, C. Jeynes, M. Tosaki, Accurate simulation of backscattering spectra in the presence of sharp resonances, Nucl Instrum Meth B, 247 (2006) 381389.

[31] A. Wiebert, B. Erlandsson, R. Hellborg, K. Stenstrom, G. Skog, THE PRESSURE PROFILE IN THE LUND-PELLETRON ACCELERATOR, Nucl Instrum Meth A, 364 (1995) 201-204.

[32] J.R. Cameron, Elastic Scattering of Alpha-Particles by Oxygen, Phys Rev, 90 (1953) 839-844.

[33] J.D. MacArthur, H.C. Evans, J.R. Leslie, H.B. Mak, 1st two Members of the Kp = OBand in Ne 20, Phys Rev C, 22 (1980) 356-361.

[34] Z.L. Wang, J.F.M. Westendorp, F.W. Saris, An Investigation of Oxygen Indiffusion during Laser Cleaning Annealing of Silicon by Means of the O-16(Alpha,Alph-O)O-16 Resonance Scattering, Nuclear Instruments \& Methods, 211 (1983) 193-201.

[35] R.A. Jarjis, On the Investigation of Alpha-Particle Resonance Elastic-Scattering from the O-16 Nucleus, Nucl Instrum Meth B, 12 (1985) 331-333.

[36] J.A. Leavitt, L.C. Mcintyre, M.D. Ashbaugh, J.G. Oder, Z. Lin, B. Dezfoulyarjomandy, Cross-Sections for 170.5-Degrees Backscattering of He-4 from Oxygen for He-4 Energies between 1.8 and 5.0 Mev, Nucl Instrum Meth B, 44 (1990) 260-265.

[37] H.S. Cheng, H. Shen, J.Y. Tang, F. Yang, Cross-Sections for 170-Degrees Backscattering of He-4 from Oxygen in the Energy-Range of 2.0-9.0 Mev, Nucl Instrum Meth B, 83 (1993) 449-453.

[38] V.I. Soroka, M.V. Artsimovich, I.Y. Lobach, I.F. Mogilnik, V.N. Pavlovich, V.V. Tokarevsky, E.M. Kudriavtsev, B.N. Romanjuk, Peculiarities of the O16(Alpha,Alpha)O-16 3.045 Mev Resonance Scattering and Its Application to Investigation of Oxygen in Silicon, Nucl Instrum Meth B, 83 (1993) 311-318. 
[39] J. Demarche, G. Terwagne, Precise measurement of the differential cross section from the $160(\mathrm{a}, \mathrm{a}) 160$ elastic reaction at 165 degrees and 170 degrees between 2.4 and 6.0 MeV, J Appl Phys, 100 (2006).

[40] A.F. Gurbich, Evaluation of non-Rutherford cross sections for IBA: Theory and results, Nucl Instrum Meth B, 261 (2007) 401-404.

[41] D.R. Tilley, C.M. Cheves, C.H. Kelley, S. Raman, H.R. Weller, Energy levels of light nuclei, A=20, Nucl Phys A, 636 (1998) 249-364.

[42] F. Michel, G. Reidemeister, Y. Kondo, A potential deduced from low energy $160(a, a)$ elastic-scattering, Phys Rev C, 51 (1995) 3290-3303.

[43] M. Mayer, Statistical analysis of cross-section data for C-12(He-4,He-4)C-12 backscattering, Nucl Instrum Meth B, 285 (2012) 116-124.

[44] E.V. Gai, A.F. Gurbich, Evaluated $12 \mathrm{C}(4 \mathrm{He}, 4 \mathrm{He}) 12 \mathrm{C}$ cross-section and its uncertainty, Nucl Instrum Meth B, 296 (2013) 87-91. 